\title{
High Frequency Peakers: Young radio sources or flaring blazars?^
}

\author{
S. Tinti ${ }^{1}$, D. Dallacasa ${ }^{2,3}$, G. De Zotti ${ }^{4,1}$, A. Celotti ${ }^{1}$, and C. Stanghellini ${ }^{3,5}$ \\ ${ }^{1}$ SISSA/ISAS, via Beirut 4, 34014 Trieste, Italy \\ e-mail: tinti@sissa.it \\ 2 Dipartimento di Astronomia, via Ranzani 1, 40127 Bologna, Italy \\ 3 Istituto di Radioastronomia - CNR, via Gobetti 101, 40129 Bologna, Italy \\ 4 INAF, Osservatorio Astronomico, Vicolo dell'Osservatorio 5, 35122 Padova, Italy \\ 5 Istituto di Radioastronomia - CNR, CP 169, 96017 Noto (SR), Italy
}

Received 8 July 2004 / Accepted 26 October 2004

\begin{abstract}
We present new, simultaneous, multifrequency observations of 45 out of the 55 candidate High Frequency Peakers (HFP) selected by Dallacasa et al. (2000), carried out 3 to 4 years after a first set of observations. Our sub-sample consists of 10 galaxies, 28 stellar objects ("quasars") and 7 unidentified sources. Both sets of observations are sensitive enough to allow the detection of variability at the $10 \%$ level or lower. While galaxies do not show significant variability, most quasars do. Seven of them no longer show the convex spectrum which is the defining property of Gigahertz Peaked Spectrum (GPS)/HFP sources and are interpreted as blazars caught by Dallacasa et al. (2000) during a flare, when a highly self-absorbed component dominated the emission. In general, the variability properties (amplitude, timescales, correlation between peak luminosity and peak frequency of the flaring component) of the quasar sub-sample resemble those of blazars. We thus conclude that most HFP candidates identified with quasars may well be flaring blazars.
\end{abstract}

Key words. galaxies: active - galaxies: quasars: general - radio continuum: galaxies

\section{Introduction}

The Compact Steep Spectrum (CSS) and Gigahertz Peaked Spectrum sources (GPS) are two classes of intrinsically compact objects (linear size $<10-1 \mathrm{kpc}$ ) defined on the basis of their spectral properties: the overall shape is convex with turnover frequencies between $100 \mathrm{MHz}$ (CSS) and a few GHz (GPS) and the spectral index at high frequencies is steep (see O'Dea 1998 , for a complete review).

The currently accepted model (the youth scenario) relates the small linear size of GPS/CSS sources to their age, implying that they are the progenitors of extended radio sources. Both kinematic studies (Owsianik \& Conway 1998; Owsianik et al. 1998) and spectral analysis (Murgia et al. 1999) indicate ages of $10^{3}-10^{5} \mathrm{yr}$. On the other hand, various observations across the electromagnetic spectrum (e.g. Fanti et al. 2000) failed to reveal the presence of a particularly dense medium that could prevent the expansion of the radio lobes in the intergalactic medium, as proposed in the frustration model (van Breugel 1984; Baum et al. 1990). So far no evidence of differences in the properties of the host galaxies between GPS/CSS and FRII radio sources has been reported (e.g. see Fanti et al. 2000).

* Appendix A is only available in electronic form at http://www . edpsciences.org
Observational studies of the population of GPS/ CSS sources have led to the discovery of an anti-correlation between the radio turnover frequency, $v_{\mathrm{p}}$, and the projected angular size, $\theta$ (O'Dea \& Baum 1997; Fanti et al. 2002). This relationship is expected if the peaked component is due to synchrotron self-absorption, where $\theta^{2} \propto v_{\mathrm{p}}^{-5 / 2}$, although free-free absorption could also play a role (Bicknell et al. 1997). In the youth scenario this correlation means that the youngest objects have the highest turnover frequencies and that the peak frequency is expected to move towards lower frequencies as the source expands and the energy density decreases. In principle, the highest the turnover frequency, the youngest the radio source is.

The shape of the radio spectrum and the position of the turnover have been used as selection tools for this class of objects. Dallacasa et al. (2000) selected a sample of "bright" radio sources with turnover frequencies above $5 \mathrm{GHz}$ and called them High Frequency Peakers (HFPs). An early summary of their properties can be found in Dallacasa (2003). Simultaneous multifrequency VLA observations were made to define the spectral shape of all candidates. The comparison with literature data showed that flux density variability is not uncommon among the candidates and that also beamed radio sources like blazars can meet the selection criteria of the sample, e.g. when 
a flaring, strongly self-absorbed synchrotron component dominates the emission spectrum. A substantial contamination of the HFP sample by blazars may obviously lead astray analyses of HFP properties.

In this paper we present the results of a second set of simultaneous multifrequency VLA observations of the sample of bright HFPs, aimed at discriminating between bonafide HFPs and blazars, and at studying the spectral evolution of the sources. At 1.465 and $1.665 \mathrm{GHz}$, longer observations were carried out to search for extended emission around the point-like dominant component. It is known that a significant fraction $(\sim 10 \%)$ of GPS sources do have extended emission (e.g. Stanghellini 2003). Also polarization data have been taken and will be presented elsewhere.

This paper is structured in the following way: Sect. 2 describes the radio properties of the sample, the second-epoch VLA observations and the data reduction; Sect. 3 presents the analysis of simultaneous radio spectra; Sect. 4 deals with the extended emission; in Sect. 5 the variability properties are investigated; the main conclusions are summarized and discussed in Sect. 6. Throughout this paper, we adopt $H_{0}=$ $50 \mathrm{~km} \mathrm{~s}^{-1} \mathrm{Mpc}^{-1}$ and $\Omega=1$

\section{The bright HFP sample}

\subsection{Selection and radio properties of the sample}

The bright HFP sample of Dallacasa et al. (2000) was selected by cross-correlating the $87 \mathrm{~GB}$ (Gregory et al. 1996) sources with $S_{4.9 \mathrm{GHz}} \geq 300 \mathrm{mJy}$ with the NVSS catalogue (Condon et al. 1998) at $1.4 \mathrm{GHz}$ and picking out those with inverted spectra $\left(\alpha<-0.5, S \propto v^{-\alpha}\right)$. Since the 87GB and the NVSS surveys were carried out at different epochs, this sample is contaminated by flat-spectrum sources that happened to be flaring when they were observed at $4.9 \mathrm{GHz}$. The sample was then "cleaned" by means of simultaneous multifrequency VLA observations at 1.365, 1.665, 4.535, 4.985, 8.085, 8.485, 14.96 and $22.46 \mathrm{GHz}$, leaving 55 sources whose single-epoch radio spectrum peaks at frequencies ranging from a few $\mathrm{GHz}$ to about $22 \mathrm{GHz}$. These "first epoch" observations were carried out in 1998-1999.

The final sample of HFP candidates comprises 11 galaxies (including a type 1 Seyfert), 36 quasars, and 8 still unidentified sources (Dallacasa et al. 2002a). For comparison, galaxies and quasars are almost equally represented in GPS samples (Stanghellini et al. 1998) and most CSS sources are galaxies (Dallacasa 2002b,c). 36 objects out of 55 have known redshift and their distribution (Fig. 1) confirms the trend of quasars and galaxies to have barely overlapping redshift ranges. The redshift distribution of quasars shows a much slower decline with increasing redshift at $z>2$ than flat-spectrum quasars in the $2.7 \mathrm{GHz}$ Parkes quarter Jansky sample (Jackson et al. 2002) with a similar flux-density limit (although at a lower frequency). This difference can be due, at least partly, to the more favorable K-correction for high- $z$ HFPs associated with their inverted spectra, and to the increasing variability amplitude with increasing frequency (Impey \& Neugebauer 1988;

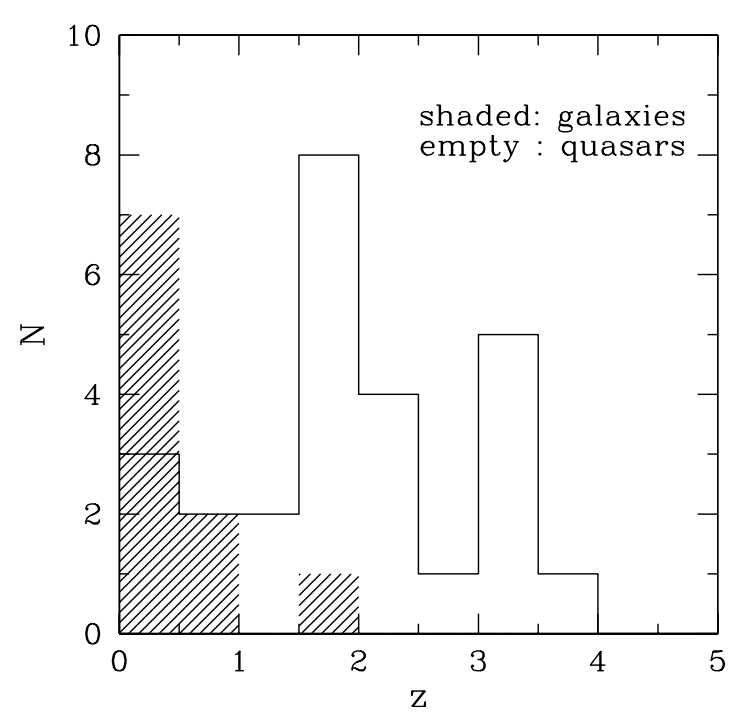

Fig. 1. Distribution of measured redshifts for the sample by Dallacasa et al. (2000).

Ciaramella et al. 2004), which also enhances the visibility of distant quasars.

\subsection{Observations and data reduction}

In July 2002 we carried out multifrequency radio observations of the 45 (out of 55) candidate HFPs that were visible during the allocated observing time. We used the VLA in the B configuration, with a frequency coverage similar to that used for the sample definition. This sub-sample comprises 10 galaxies (including the type 1 Seyfert), 28 stellar objects, henceforth referred to as "quasars" (including a BL Lac), and 7 unidentified sources. We used the intermediate frequencies (IFs) of 1.465 and $1.665 \mathrm{GHz}$ in the $\mathrm{L}$ band, of 4.565 and $4.935 \mathrm{GHz}$ in the $\mathrm{C}$ band, and of 8.085 and $8.465 \mathrm{GHz}$ in the $\mathrm{X}$ band, while the standard VLA frequencies were used in the $\mathrm{U}$ band $(14.96 \mathrm{GHz})$ and in the $\mathrm{K}$ band $(22.46 \mathrm{GHz})$. The observing bandwidth was chosen to be $50 \mathrm{MHz}$ per IF. Separate analysis for each IF in L, C and $\mathrm{X}$ bands was carried out in order to improve the spectral coverage of the data.

Each source was observed typically for $1 \mathrm{~min}$ in $\mathrm{C}, \mathrm{X}, \mathrm{U}$ and $\mathrm{K}$ bands, and for 9 min in $\mathrm{L}$ band in a single snapshot, cycling through frequencies. The flux density measurements can thus be considered as simultaneous.

Two scans were spent on the primary flux density calibrator 3C 286, which was used also for the calibration of the absolute orientation of the polarization vector. Secondary calibrators were observed for $1.5 \mathrm{~min}$ at each frequency about every $25 \mathrm{~min}$; they were chosen aiming at minimizing the telescope slewing time. Accurate positions of the target sources were obtained from the JVAS catalogue (Patnaik et al. 1992; Browne et al. 1998; Wilkinson et al. 1998).

The data reduction followed the standard procedures for the VLA, implemented in the NRAO AIPS software. The L-band imaging has been quite complicated since a number of confusing sources fall within the primary beam, and an accurate flux density measurement could be obtained only once the 
confusing sources had been cleaned out. Generally, at least one iteration of phase-only self-calibration has been performed before the final imaging, although a few sources required several iterations. Some radio frequency interferences affected the $1.665 \mathrm{GHz}$ data, so that a few sources could not be imaged and flux densities could not be derived at this frequency. A Gaussian fit was performed on the final image by means of the task JMFIT, and the flux densities of the extended sources were determined with TVSTAT and IMSTAT.

The L band observations by Dallacasa et al. (2000) were not adequate to reveal possible extended emission given that were taken with the VLA in various configurations and were too short (1 min). The present deeper exposures detected extended emission for a considerable fraction of sources (see Sect. 4).

The rms noise levels in the image plane are relevant only for flux densities of a few mJy and are generally consistent with the expected thermal noise. For our bright sources the main uncertainty comes from the amplitude calibration error, conservatively estimated to be $(1 \sigma) 3 \%$ for the $\mathrm{L}, \mathrm{C}$ and $\mathrm{X}$ bands, $5 \%$ for the $\mathrm{U}$ band, and $10 \%$ for the $\mathrm{K}$ band.

The new multifrequency measurements are listed in Cols. 6-13 of Table 1, together with the J2000 name (Col. 1), the optical identification and the redshift taken from the NED database (Cols. 2 and 3, respectively), the $4.9 \mathrm{GHz}$ flux density (Col. 4) measured by Dallacasa et al. (2000); the labels C and E in Col. 5 stand for compact or extended sources respectively, as seen in the L band images (see Sect. 4, Appendix).

The time-lag between the the present data-set and the previous observations is of about 3 to 4 years. The years of observations of each source are specified in Table 2.

\section{Spectral analysis}

In order to estimate the peak flux densities, $S_{\mathrm{p}}$, and frequencies, $v_{\mathrm{p}}$, of the sources, we have fitted the simultaneous radio spectra at the two epochs using an hyperbolic function (Dallacasa et al. 2000) of the form:

$\log (S)=a-\left[b^{2}+(c \log v-d)^{2}\right]^{1 / 2}$

with the optically thin and thick spectral indices as asymptotes. The parameters of this function are related to $S_{\mathrm{p}}$ and $v_{\mathrm{p}}$ $\left[\log \left(v_{\mathrm{p}}\right)=d / c, \log \left(S_{\mathrm{p}}\right)=a-b\right]$, so that Eq. (1) can be rewritten as:

$\log (S)=\log \left(S_{\mathrm{p}}\right)+b-\left[b^{2}+c^{2}\left(\log v-\log v_{\mathrm{p}}\right)^{2}\right]^{1 / 2}$.

The best fit values of $v_{\mathrm{p}}$ and $S_{\mathrm{p}}$, obtained minimizing the chisquare function with the Minuit package (CERN libraries), are reported, with their errors, in Table 2 . The radio spectra of all sources are shown in Fig. 2 where the stars and the filled circles refer to the first and to the second epoch of simultaneous multifrequency VLA data, respectively, while the dashed and solid lines indicate the corresponding fits.

A Kolmogorov-Smirnov (KS) test did not detect any significant difference among the distributions of the observed turnover frequencies (shown in Fig. 3) and of the peak flux densities at the two epochs. This is not surprising since the time-lag is too short for an evolution of source spectra (see Sect. 5) to be detected.

The observed peak frequency distributions of candidate HFP galaxies and quasars are similar (left-hand panel of Fig. 4) as a consequence of the selection criterion, but quasars are found up to much higher redshifts (Fig. 1), implying much higher values of $v_{\mathrm{p}}$ in the source frame (Fig. 4, right-hand panel). A similar behavior is found for GPS sources (Snellen 1997; Stanghellini et al. 1998), whose peak frequencies are, on average, about a factor of 5 lower.

\section{Extended emission}

Within our sub-sample, 14 (31\%) sources show some amount of extended emission ( 2 galaxies, 9 quasars and 3 objects without optical identification) on scales ranging from 6 to 35 arcsec. In the Appendix we comment on such sources, based on our own observations and on published VLBA/VLA data. Our images are presented in Figs. A1-A10. The flux density of the extended emission has been measured as the difference between the integrated flux density over the whole source extension and the flux density obtained using a point source model (see Table 3). Weak emission below the surface brightness limit $(\sim 0.2 \mathrm{mJy} /$ beam $)$ of our maps cannot be excluded.

The total projected linear size of all these sources exceeds the classical definition of a "compact" radio source, i.e. about 15-20 kpc (O'Dea 1998). The total flux density of the extended emission ranges between a few mJy and $80 \mathrm{mJy}$, with $1.4 \mathrm{GHz}$ luminosities ranging from $\simeq 10^{24} \mathrm{~W} / \mathrm{Hz}$ (close to the upper end of the range for FR I sources) to $\simeq 10^{27} \mathrm{~W} / \mathrm{Hz}$, well within the luminosity range of FR II sources. The unresolved to extended luminosity ratios are in the range 4 to 78 .

The fraction of sources with extended emission $(\simeq 30 \%)$ is larger than in GPS samples, where it is found to be $\simeq 10 \%$ (Stanghellini et al. 1990; Baum et al. 1990; Stanghellini et al., in preparation). However, no dependence of the fraction of sources with extended emission on peak frequency can be found within our sample: if we split it into two roughly equally populated sub-samples (with $v_{\mathrm{p}}>5.5 \mathrm{GHz}$ and $v_{\mathrm{p}}<5.5$, $v_{\mathrm{p}}$ being the second epoch peak frequency in the observer's frame), we have approximately the same fraction of sources with extended emission in each.

The presence of radio emission on scales of tens of $\mathrm{kpc}$ or even larger can indicate that we are really dealing with an evolved source but can also be reconciled with the youth scenario under the hypothesis of recurrent activity proposed by Baum et al. (1990), whereby a newly born source is propagating amidst the relic of previous large scale radio activity. At low frequencies (hundreds of $\mathrm{MHz}$ ) the extended emission should be dominant, because of its steep spectrum.

Seven out of the nine quasars with extended emission have strongly variable spectra. Five of them did not show anymore a convex spectrum (see Sect. 5) when were re-observed, and therefore are not HFPs. On the other hand, the two galaxies with some amount of resolved emission on a kpc scale showed, at both epochs, the typical peaked spectrum. One is the well known J0111 + $3906($ B0108 + 388) for which Baum et al. (1990) worked out the recurrent activity hypothesis. We are 
Table 1. Integrated flux densities of candidates HFP sources at the 2002 epoch.

\begin{tabular}{|c|c|c|c|c|c|c|c|c|c|c|c|c|}
\hline $\begin{array}{l}\text { J2000 } \\
\text { Name }\end{array}$ & ID & $z$ & $\begin{array}{l}S_{5.0}^{\text {old }} \\
\text { mJy }\end{array}$ & & $\begin{array}{l}S_{1.4} \\
\mathrm{mJy}\end{array}$ & $\begin{array}{l}S_{1.7} \\
\mathrm{mJy}\end{array}$ & $\begin{array}{l}S_{4.5} \\
\mathrm{mJy}\end{array}$ & $\begin{array}{l}S_{4.9} \\
\mathrm{mJy}\end{array}$ & $\begin{array}{l}S_{8.1} \\
\mathrm{mJy}\end{array}$ & $\begin{array}{l}S_{8.5} \\
\mathrm{mJy}\end{array}$ & $\begin{array}{r}S_{15.0} \\
\mathrm{mJy} \\
\end{array}$ & $\begin{array}{r}S_{22.5} \\
\mathrm{mJy} \\
\end{array}$ \\
\hline $0003+2129$ & $\mathrm{G}^{*}$ & 0.4 & 265 & $\mathrm{C}$ & 102 & 120 & 250 & 253 & 234 & 227 & 140 & 86 \\
\hline $0005+0524$ & $\mathrm{Q}$ & 1.887 & 229 & $\mathrm{C}$ & 168 & 186 & 220 & 213 & 171 & 166 & 111 & 82 \\
\hline $0037+0808$ & G? & $>1.8$ & 292 & $\mathrm{C}$ & 101 & 118 & 281 & 283 & 267 & 262 & 190 & 143 \\
\hline $0111+3906$ & G & 0.668 & 1324 & $\mathrm{E}$ & 476 & 594 & 1313 & 1286 & 978 & 937 & 507 & 315 \\
\hline $0116+2422$ & & & 243 & $\mathrm{C}$ & 110 & 124 & 254 & 258 & 252 & 248 & & 149 \\
\hline $0217+0144$ & Q & 1.715 & 1862 & $\mathrm{E}$ & 810 & 802 & 743 & 745 & 810 & 810 & 856 & 838 \\
\hline $0329+3510$ & $\mathrm{Q}^{*}$ & & 770 & $\mathrm{E}$ & 442 & 462 & 560 & 555 & 608 & 613 & 659 & 702 \\
\hline $0357+2319$ & $\mathrm{Q}^{*}$ & & 560 & $\mathrm{C}$ & 139 & 133 & 131 & 130 & 143 & 144 & 154 & 168 \\
\hline $0428+3259$ & $\mathrm{G}^{*}$ & 0.3 & 506 & $\mathrm{E}$ & 177 & 202 & 493 & 506 & 525 & 514 & 375 & 263 \\
\hline $0519+0848$ & & & 278 & $\mathrm{E}$ & 279 & 296 & 446 & 446 & 435 & 430 & 420 & 401 \\
\hline $0625+4440$ & BL & & 442 & $\mathrm{C}$ & 172 & 184 & 231 & 231 & 238 & 238 & 219 & 210 \\
\hline $0638+5933$ & & & 591 & $\mathrm{C}$ & 277 & 301 & 590 & 606 & 668 & 667 & 620 & 567 \\
\hline $0642+6758$ & Q & 3.180 & 474 & $\mathrm{C}$ & 245 & 288 & 429 & 417 & 331 & 321 & 203 & 149 \\
\hline $0646+4451$ & Q & 3.396 & 1896 & $\mathrm{C}$ & 522 & 612 & 2580 & 2770 & 3709 & 3757 & 3691 & 3318 \\
\hline $0650+6001$ & Q & 0.455 & 1236 & $\mathrm{C}$ & 517 & 625 & 1142 & 1136 & 989 & 964 & 671 & 495 \\
\hline $1335+4542$ & Q & 2.449 & 735 & $\mathrm{C}$ & 294 & 366 & 797 & 785 & 613 & 592 & 359 & 234 \\
\hline $1335+5844$ & & & 723 & $\mathrm{C}$ & 319 & 396 & 734 & 725 & 680 & 671 & 531 & 253 \\
\hline $1407+2827$ & G & 0.0769 & 2362 & $\mathrm{C}$ & 910 & 1100 & 2469 & 2463 & 2114 & 2050 & 1139 & 604 \\
\hline $1412+1334$ & & & 330 & $\mathrm{C}$ & 205 & 236 & 345 & 337 & 275 & 267 & 185 & 130 \\
\hline $1424+2256$ & Q & 3.626 & 607 & $\mathrm{C}$ & 352 & 414 & 686 & 669 & 479 & 460 & 251 & 145 \\
\hline $1430+1043$ & Q & 1.710 & 910 & $\mathrm{C}$ & 326 & 402 & 885 & 882 & 770 & 752 & 546 & 385 \\
\hline $1457+0749$ & & & 241 & $\mathrm{E}$ & 360 & 367 & 262 & 251 & 194 & 188 & 141 & 109 \\
\hline $1505+0326$ & Q & 0.411 & 929 & $\mathrm{C}$ & 413 & 465 & 740 & 741 & 721 & 710 & 665 & 567 \\
\hline $1511+0518$ & Sy1 & 0.084 & 536 & $\mathrm{C}$ & 90 & 115 & 568 & 607 & 848 & 861 & 843 & 617 \\
\hline $1526+6650$ & $\mathrm{Q}$ & 3.02 & 411 & $\mathrm{C}$ & 115 & 145 & 408 & 410 & 352 & 341 & 193 & 107 \\
\hline $1603+1105$ & & & 270 & $\mathrm{E}$ & 182 & 188 & 214 & 214 & 225 & 225 & 234 & 217 \\
\hline $1616+0459$ & Q & 3.197 & 892 & $\mathrm{C}$ & 317 & 382 & 787 & 771 & 582 & 559 & 333 & 212 \\
\hline $1623+6624$ & G & 0.203 & 298 & $\mathrm{C}$ & 159 & 175 & 295 & 298 & 287 & 283 & 224 & 175 \\
\hline $1645+6330$ & Q & 2.379 & 513 & $\mathrm{C}$ & 303 & 315 & 510 & 526 & 615 & 618 & 596 & 493 \\
\hline $1735+5049$ & $\mathrm{G} ?$ & & 968 & $\mathrm{C}$ & 448 & & 925 & 943 & 934 & 920 & 740 & 587 \\
\hline $1800+3848$ & Q & 2.092 & 791 & $\mathrm{E}$ & 313 & 331 & 702 & 748 & 1044 & 1063 & 1174 & 1076 \\
\hline $1811+1704$ & $\mathrm{Q}^{*}$ & & 691 & $\mathrm{E}$ & 545 & 530 & 494 & 491 & 508 & 509 & 499 & 418 \\
\hline $1840+3900$ & $\mathrm{Q}$ & 3.095 & 203 & $\mathrm{C}$ & 125 & 130 & 169 & 167 & 161 & 158 & 134 & 114 \\
\hline $1850+2825$ & Q & 2.560 & 1246 & $\mathrm{C}$ & 203 & 223 & 1096 & 1185 & 1540 & 1541 & 1318 & 1045 \\
\hline $1855+3742$ & $\mathrm{G}^{*}$ & 0.5 & 364 & $\mathrm{C}$ & 193 & 191 & 362 & 345 & 222 & 212 & 124 & 91 \\
\hline $2021+0515$ & $\mathrm{Q}^{*}$ & & 477 & $\mathrm{C}$ & 260 & 302 & 450 & 441 & 377 & 368 & 267 & 191 \\
\hline $2024+1718$ & S & $1.050 ?$ & 572 & $\mathrm{E}$ & 280 & 304 & 676 & 704 & 803 & 800 & 697 & 569 \\
\hline $2101+0341$ & Q & 1.013 & 920 & $\mathrm{E}$ & 553 & 610 & 694 & 678 & 593 & 583 & 499 & 478 \\
\hline $2123+0535$ & Q & 1.878 & 1896 & $\mathrm{E}$ & 1954 & 2006 & 2084 & 2111 & 2462 & 2482 & 2755 & 2560 \\
\hline $2203+1007$ & $\mathrm{G}^{*}$ & 0.9 & 319 & $\mathrm{C}$ & 124 & 123 & 312 & 306 & 243 & 234 & 129 & 77 \\
\hline $2207+1652$ & $\mathrm{Q}^{*}$ & & 551 & $\mathrm{E}$ & 223 & & 249 & 247 & 227 & 223 & 188 & 163 \\
\hline $2212+2355$ & $\mathrm{~S}$ & & 1182 & $\mathrm{C}$ & 601 & & 963 & 979 & 1033 & 1028 & 975 & 915 \\
\hline $2257+0243$ & Q & 2.081 & 274 & $\mathrm{C}$ & 174 & & 274 & 292 & 439 & 450 & 558 & 528 \\
\hline $2320+0513$ & Q & 0.622 & 1196 & $\mathrm{E}$ & 640 & & 657 & 656 & 720 & 725 & 806 & 843 \\
\hline $2330+3348$ & Q & 1.809 & 558 & $\mathrm{C}$ & 370 & 383 & 407 & 407 & 457 & 463 & 532 & 548 \\
\hline
\end{tabular}

${ }^{*}$ Indicates the sources optically identified by Dallacasa et al. (2002a). 
Table 2. Peak frequencies and flux densities.

\begin{tabular}{|c|c|c|c|c|c|c|c|c|c|c|}
\hline \multirow[t]{2}{*}{ Name } & & & \multicolumn{2}{|c|}{1998} & \multicolumn{2}{|c|}{1999} & \multicolumn{2}{|c|}{2002} & \multirow[t]{2}{*}{ V } & \multirow[t]{2}{*}{$\Delta v /\left(v_{\mathrm{p}}^{I} \cdot \Delta t\right)$} \\
\hline & & & $\begin{array}{l}S_{\mathrm{p}} \\
\mathrm{mJy}\end{array}$ & $\begin{array}{l}v_{\mathrm{p}} \\
\mathrm{GHz}\end{array}$ & $\begin{array}{l}S_{\mathrm{p}} \\
\mathrm{mJy}\end{array}$ & $\begin{array}{l}v_{\mathrm{p}} \\
\mathrm{GHz}\end{array}$ & $\begin{array}{l}S_{\mathrm{p}} \\
\mathrm{mJy}\end{array}$ & $\begin{array}{l}v_{\mathrm{p}} \\
\mathrm{GHz}\end{array}$ & & \\
\hline $0003+2129$ & $\mathrm{G}^{*}$ & $\mathrm{C}$ & $272 \pm 5$ & $5.7 \pm 0.1$ & & & $257 \pm 5$ & $5.4 \pm 0.1$ & 3.02 & $0.018 \pm 0.009$ \\
\hline $0005+0524$ & $\mathrm{Q}$ & $\mathrm{C}$ & & & $235 \pm 6$ & $4.13 \pm 0.09$ & $228 \pm 7$ & $3.40 \pm 0.09$ & 3.18 & $0.170 \pm 0.03$ \\
\hline $0037+0808$ & $\mathrm{G} ?$ & $\mathrm{C}$ & & & $287 \pm 5$ & $5.9 \pm 0.1$ & $287 \pm 6$ & $6.2 \pm 0.2$ & 0.46 & $-0.05 \pm 0.04$ \\
\hline $0111+3906$ & $\mathrm{G}$ & $\mathrm{E}$ & & & $1333 \pm 28$ & $4.76 \pm 0.06$ & $1303 \pm 28$ & $4.68 \pm 0.07$ & 0.46 & $0.01 \pm 0.01$ \\
\hline $0116+2422$ & & $\mathrm{C}$ & $245 \pm 4$ & $5.1 \pm 0.1$ & & & $266 \pm 9$ & $6.3 \pm 0.3$ & 5.59 & \\
\hline $0217+0144$ & Q & $\mathrm{E}$ & & & $2557 \pm 105$ & $18 \pm 3$ & & flat & 238.27 & \\
\hline $0329+3510$ & $\mathrm{Q}^{*}$ & $\mathrm{E}$ & $768 \pm 13$ & $6.7 \pm 0.3$ & & & & flat & 21.60 & \\
\hline $0357+2319$ & $\mathrm{Q}^{*}$ & $\mathrm{C}$ & $637 \pm 15$ & $12 \pm 1$ & & & & flat & 420.12 & \\
\hline $0428+3259$ & $\mathrm{G}^{*}$ & $\mathrm{E}$ & $545 \pm 10$ & $7.3 \pm 0.2$ & & & $539 \pm 11$ & $6.8 \pm 0.2$ & 1.20 & $0.02 \pm 0.01$ \\
\hline $0519+0848$ & & $\mathrm{E}$ & $>380$ & $>22$ & $>560$ & $>22$ & $448 \pm 7$ & $7.4 \pm 0.6$ & 62.80 & \\
\hline $0625+4440$ & $\mathrm{BL}$ & $\mathrm{C}$ & $575 \pm 17$ & $13 \pm 2$ & & & $237 \pm 4$ & $7.4 \pm 1.0$ & 160.34 & \\
\hline $0638+5933$ & & $\mathrm{C}$ & $700 \pm 17$ & $12 \pm 2$ & & & $675 \pm 13$ & $9.2 \pm 0.7$ & 0.83 & \\
\hline $0642+6758$ & $\mathrm{Q}$ & $\mathrm{C}$ & $481 \pm 11$ & $4.5 \pm 0.1$ & & & $431 \pm 10$ & $4.08 \pm 0.08$ & 6.77 & $0.10 \pm 0.03$ \\
\hline $0646+4451$ & Q & $\mathrm{C}$ & $3371 \pm 118$ & $15 \pm 2$ & & & $3982 \pm 92$ & $11.2 \pm 0.6$ & 36.91 & $0.3 \pm 0.1$ \\
\hline $0650+6001$ & Q & $\mathrm{C}$ & $1290 \pm 19$ & $7.6 \pm 0.3$ & & & $1143 \pm 27$ & $5.2 \pm 0.2$ & 19.92 & $0.11 \pm 0.01$ \\
\hline $1335+4542$ & Q & $\mathrm{C}$ & $737 \pm 18$ & $5.1 \pm 0.1$ & & & $793 \pm 17$ & $4.9 \pm 0.1$ & 1.23 & $0.03 \pm 0.02$ \\
\hline $1335+5844$ & & $\mathrm{C}$ & $730 \pm 12$ & $6.0 \pm 0.2$ & & & $736 \pm 12$ & $5.5 \pm 0.1$ & 1.50 & \\
\hline $1407+2827$ & G & $\mathrm{C}$ & $2320 \pm 36$ & $5.34 \pm 0.05$ & $2388 \pm 41$ & $5.20 \pm 0.09$ & $2484 \pm 49$ & $5.01 \pm 0.08$ & 1.77 & $0.017 \pm 0.005$ \\
\hline $1412+1334$ & & $\mathrm{C}$ & $337 \pm 7$ & $4.7 \pm 0.1$ & & & $345 \pm 8$ & $4.18 \pm 0.09$ & 1.03 & \\
\hline $1424+2256$ & Q & $\mathrm{C}$ & $623 \pm 15$ & $4.13 \pm 0.07$ & & & $698 \pm 18$ & $3.94 \pm 0.06$ & 2.11 & $0.05 \pm 0.03$ \\
\hline $1430+1043$ & Q & $\mathrm{C}$ & $905 \pm 14$ & $6.5 \pm 0.2$ & & & $887 \pm 39$ & $5.7 \pm 0.1$ & 4.19 & $0.08 \pm 0.02$ \\
\hline $1457+0749$ & & $\mathrm{E}$ & & & $239 \pm 4$ & $5.4 \pm 0.3$ & $367 \pm 12$ & $1.7 \pm 0.3$ & 39.74 & \\
\hline $1505+0326$ & $\mathrm{Q}$ & $\mathrm{C}$ & & & $937 \pm 14$ & $7.1 \pm 0.4$ & $744 \pm 11$ & $6.8 \pm 0.4$ & 19.65 & $0.02 \pm 0.04$ \\
\hline $1511+0518$ & Sy 1 & $\mathrm{C}$ & $778 \pm 18$ & $11.1 \pm 0.4$ & & & $903 \pm 20$ & $10.6 \pm 0.4$ & 8.28 & $0.01 \pm 0.01$ \\
\hline $1526+6650$ & $\mathrm{Q}$ & $\mathrm{C}$ & $427 \pm 13$ & $5.7 \pm 0.1$ & & & $417 \pm 10$ & $5.5 \pm 0.1$ & 0.53 & $0.04 \pm 0.03$ \\
\hline $1603+1105$ & & $\mathrm{E}$ & & & $277 \pm 4$ & $7.7 \pm 0.4$ & $229 \pm 9$ & $13 \pm 7$ & 16.65 & \\
\hline $1616+0459$ & $\mathrm{Q}$ & $\mathrm{C}$ & & & $897 \pm 20$ & $4.7 \pm 0.1$ & $782 \pm 17$ & $4.63 \pm 0.09$ & 15.20 & $0.02 \pm 0.04$ \\
\hline $1623+6624$ & G & $\mathrm{C}$ & $291 \pm 5$ & $6.0 \pm 0.2$ & & & $302 \pm 8$ & $6.0 \pm 0.2$ & 0.47 & $0.00 \pm 0.01$ \\
\hline $1645+6330$ & $\mathrm{Q}$ & $\mathrm{C}$ & $628 \pm 18$ & $14 \pm 2$ & & & $629 \pm 17$ & $10.1 \pm 0.7$ & 2.74 & $0.2 \pm 0.1$ \\
\hline $1735+5049$ & $\mathrm{G} ?$ & $\mathrm{C}$ & $972 \pm 18$ & $6.4 \pm 0.2$ & & & $955 \pm 16$ & $6.3 \pm 0.3$ & 0.15 & \\
\hline $1800+3848$ & $\mathrm{Q}$ & $\mathrm{E}$ & & & $1392 \pm 65$ & $17 \pm 3$ & $1226 \pm 49$ & $13 \pm 1$ & 1.91 & $0.2 \pm 0.2$ \\
\hline $1811+1704$ & $\mathrm{Q}^{*}$ & $\mathrm{E}$ & & & $809 \pm 19$ & $12 \pm 1$ & & flat & 58.74 & \\
\hline $1840+3900$ & $\mathrm{Q}$ & $\mathrm{C}$ & & & $201 \pm 3$ & $5.7 \pm 0.5$ & $169 \pm 4$ & $5.2 \pm 0.4$ & 20.16 & $0.1 \pm 0.2$ \\
\hline $1850+2825$ & Q & $\mathrm{C}$ & & & $1567 \pm 32$ & $9.1 \pm 0.3$ & $1591 \pm 32$ & $9.5 \pm 0.3$ & 4.86 & $-0.05 \pm 0.06$ \\
\hline $1855+3742$ & $\mathrm{G}^{*}$ & $\mathrm{C}$ & & & $426 \pm 42$ & $4.00 \pm 0.07$ & $423 \pm 310$ & $3.81 \pm 0.06$ & 1.32 & $0.02 \pm 0.01$ \\
\hline $2021+0515$ & $\mathrm{Q}^{*}$ & $\mathrm{C}$ & & & $497 \pm 13$ & $3.75 \pm 0.08$ & $446 \pm 10$ & $4.5 \pm 0.1$ & 5.92 & \\
\hline $2024+1718$ & $\mathrm{~S}$ & $\mathrm{E}$ & & & $845 \pm 32$ & $14 \pm 2$ & $807 \pm 16$ & $8.6 \pm 0.4$ & 9.58 & $0.26 \pm 0.06$ \\
\hline $2101+0341$ & Q & $\mathrm{E}$ & & & $1635 \pm 90$ & $17 \pm 2$ & $737 \pm 212$ & $3.7 \pm 0.2$ & 119.87 & $0.53 \pm 0.02$ \\
\hline $2123+0535$ & $\mathrm{Q}$ & $\mathrm{E}$ & & & $2392 \pm 103$ & $18 \pm 4$ & & flat & 27.49 & \\
\hline $2203+1007$ & $\mathrm{G}^{*}$ & $\mathrm{C}$ & & & $319 \pm 7$ & $4.86 \pm 0.07$ & $312 \pm 7$ & $5.0 \pm 0.1$ & 5.02 & $-0.02 \pm 0.02$ \\
\hline $2207+1652$ & $\mathrm{Q}^{*}$ & $\mathrm{E}$ & & & $572 \pm 9$ & $7.4 \pm 0.3$ & $252 \pm 7$ & $3.5 \pm 0.3$ & 228.45 & \\
\hline $2212+2355$ & $\mathrm{~S}$ & $\mathrm{C}$ & & & $1379 \pm 13$ & $13 \pm 2$ & $1031 \pm 17$ & $9 \pm 1$ & 22.62 & \\
\hline $2257+0243$ & Q & $\mathrm{C}$ & & & & $>22$ & & $>22$ & 1.85 & \\
\hline $2320+0513$ & Q & $\mathrm{E}$ & & & $1176 \pm 20$ & $5.4 \pm 0.2$ & & flat & 86.24 & \\
\hline $2330+3348$ & Q & $\mathrm{C}$ & & & $558 \pm 9$ & $5.6 \pm 0.3$ & & flat & 21.42 & \\
\hline
\end{tabular}


S. Tinti et al.: High Frequency Peakers: Young radio sources or flaring blazars?

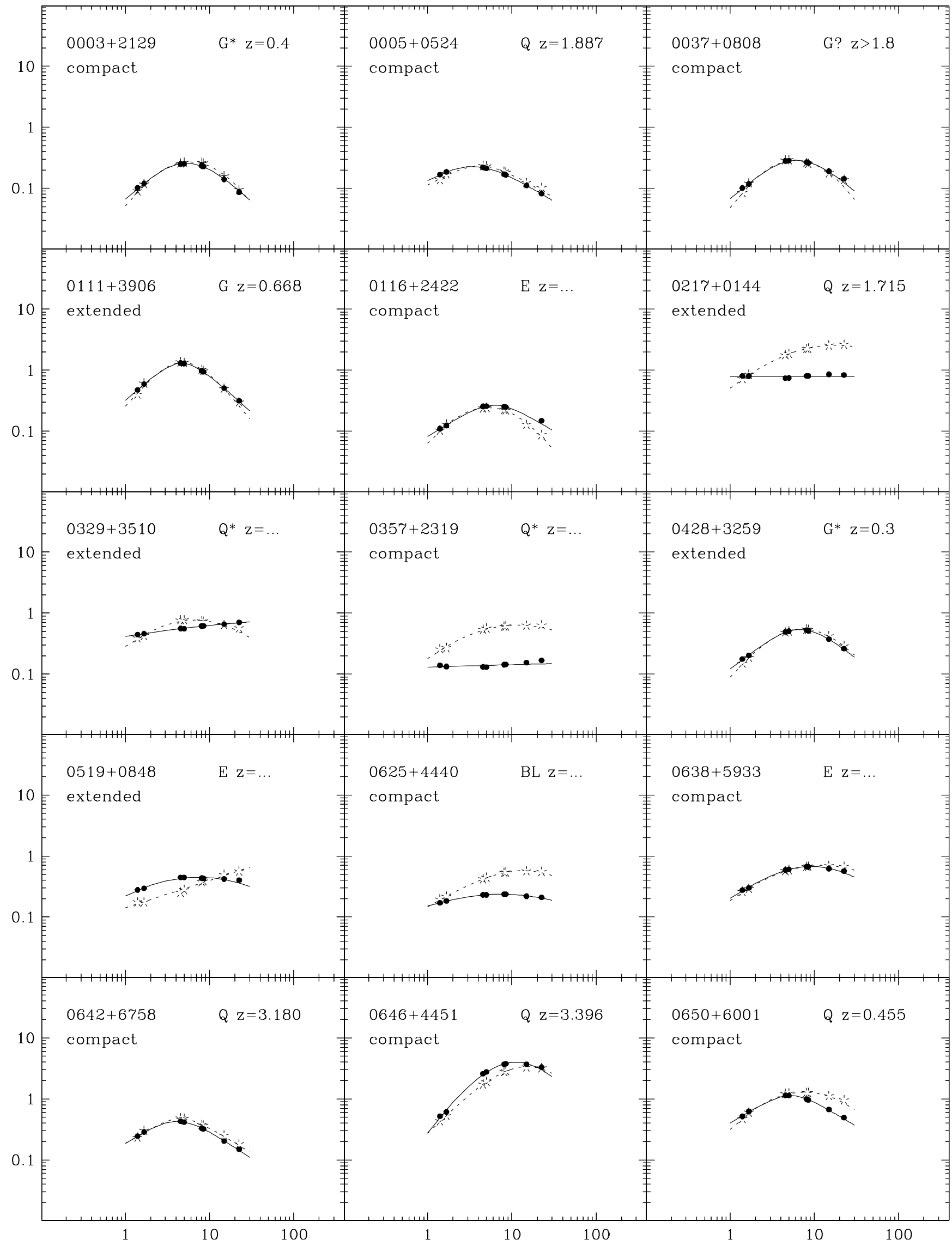

Fig. 2. Radio spectra of sources $\left[S_{v}(\mathrm{Jy})\right.$ vs. $\left.v(\mathrm{GHz})\right]$. Stars and filled circles represent the first and the second epoch of simultaneous multifrequency VLA data respectively; the dashed and solid lines show the corresponding polynomial fits. 
S. Tinti et al.: High Frequency Peakers: Young radio sources or flaring blazars?

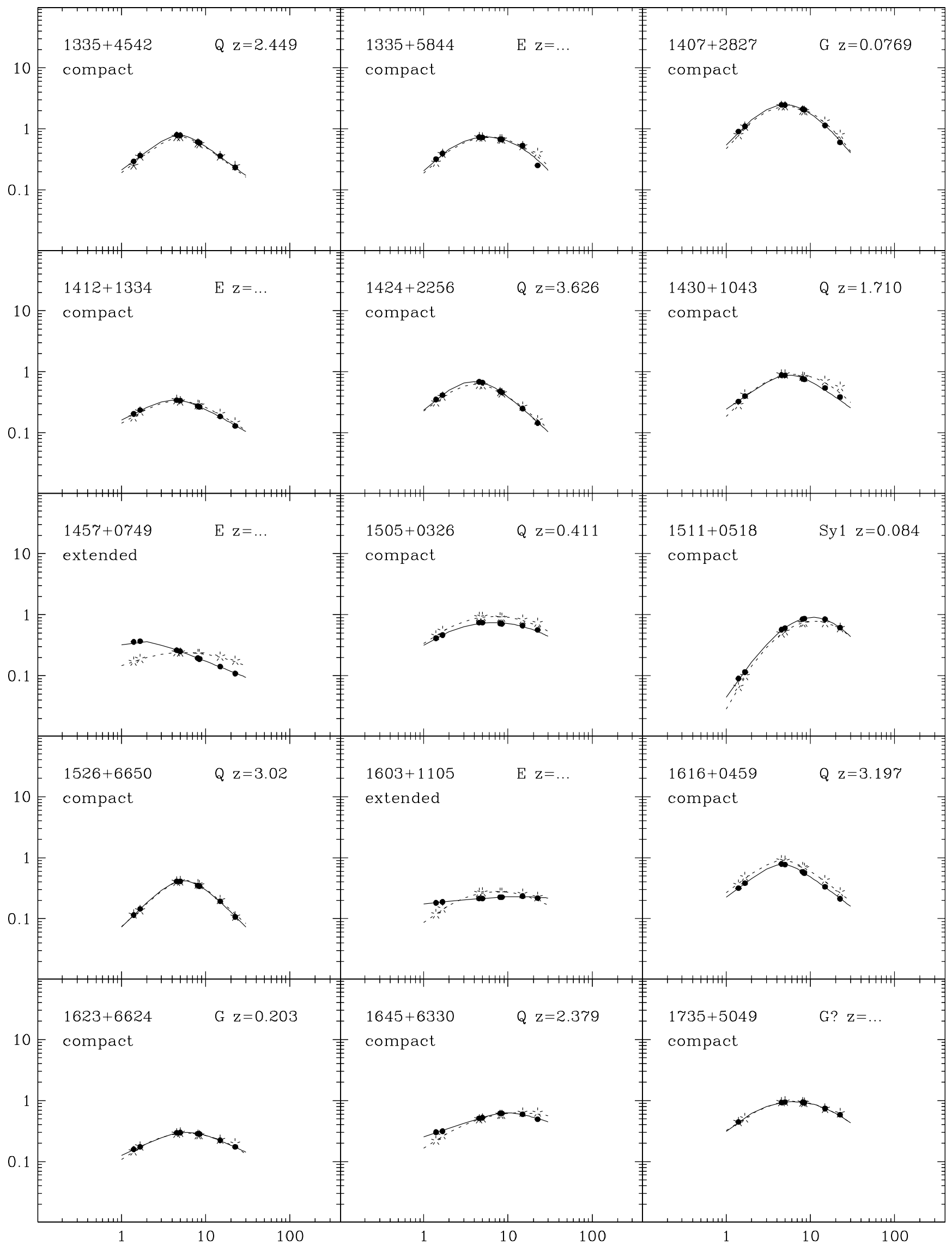

Fig. 2. continued. 
S. Tinti et al.: High Frequency Peakers: Young radio sources or flaring blazars?

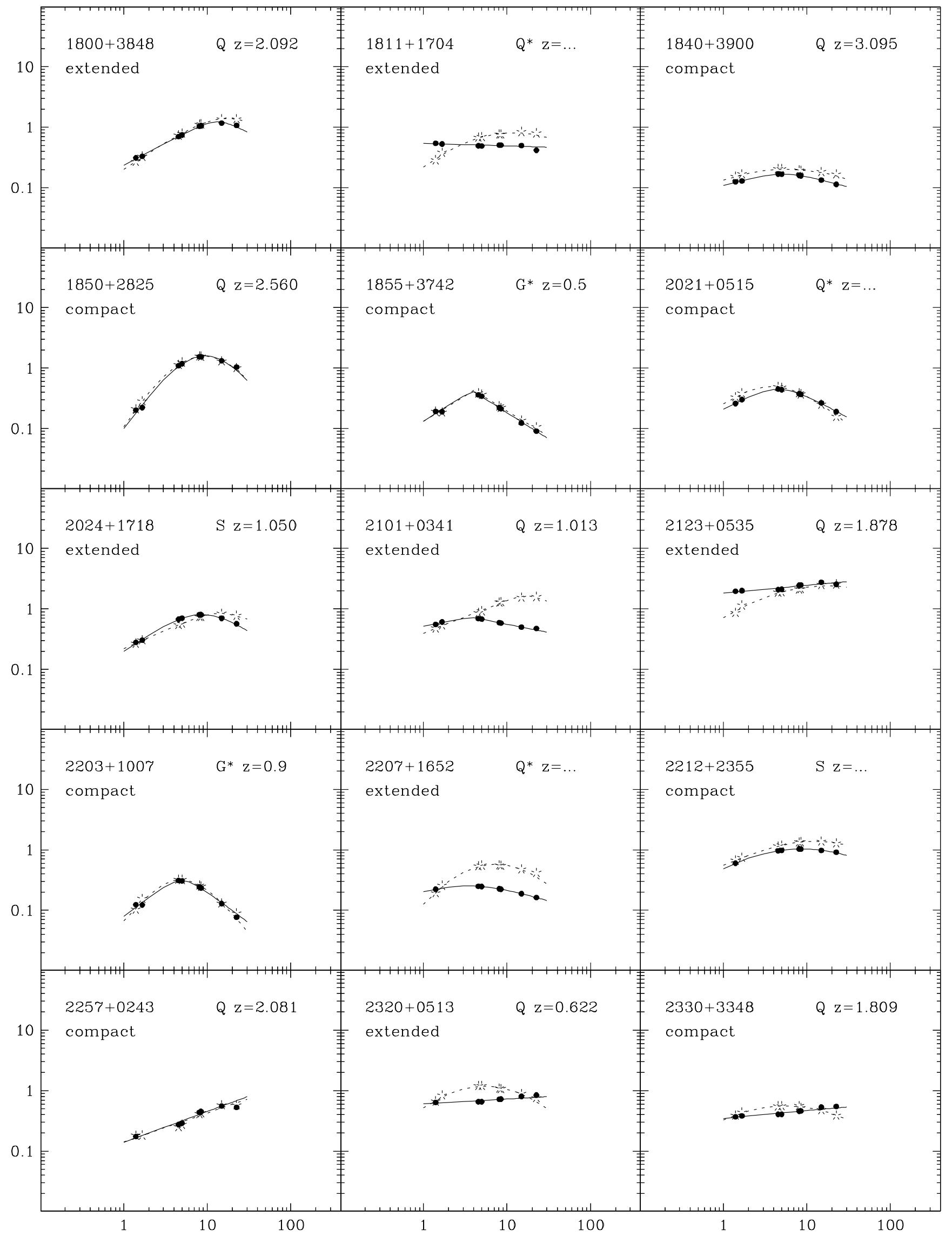

Fig. 2. continued. 
Table 3. Parameters of the extended objects.

\begin{tabular}{|c|c|c|c|c|c|c|c|c|c|}
\hline \multirow[t]{2}{*}{ Name } & \multicolumn{2}{|c|}{$S_{1.4}(\mathrm{mJy})$} & \multicolumn{2}{|c|}{$S_{1.7}(\mathrm{mJy})$} & \multicolumn{2}{|c|}{$S_{\mathrm{C} \text { band }}(\mathrm{mJy})$} & \multicolumn{2}{|c|}{$S_{\mathrm{X} \text { band }}(\mathrm{mJy})$} & \multirow[t]{2}{*}{$L S(\operatorname{arcsec})$} \\
\hline & peak & ext & peak & ext & peak & ext & peak & ext & \\
\hline J0111+3906 & 477 & 10 & 594 & 7 & & & & & 17 \\
\hline $\mathrm{J} 0217+0144$ & 750 & 75 & 766 & 59 & 736 & 20 & 808 & 8 & $>6(4.5 \mathrm{GHz})$ \\
\hline $\mathrm{J} 0329+3510$ & 423 & 80 & 455 & 65 & 553 & 5 & & & 35 \\
\hline $\mathrm{J} 0428+3259$ & 167 & 12 & 198 & 8 & 492 & 3 & & & $>8(4.5 \mathrm{GHz})$ \\
\hline J0519+0848 & 268 & 17 & 287 & 15 & 444 & 4 & & & 10 \\
\hline $\mathrm{J} 1457+0749$ & 354 & 13 & 361 & 12 & & & & & 12 \\
\hline $\mathrm{J} 1603+1105$ & 170 & 27 & 175 & 26 & 213 & 2 & & & 94 \\
\hline $\mathrm{J} 1800+3848$ & 311 & 4 & 329 & 5 & & & & & $>6$ \\
\hline $\mathrm{J} 1811+1704$ & 537 & 15 & 523 & 18 & 488 & 4 & 494 & 6 & 22 \\
\hline J2024+1718 & 279 & 12 & 304 & 10 & & & & & 11 \\
\hline $\mathrm{J} 2101+0341$ & 550 & 15 & 609 & 14 & & & & & $22(\mathrm{EW}) \times 14(\mathrm{NS})$ \\
\hline $\mathrm{J} 2123+0535$ & 1949 & 14 & 1999 & 34 & & & & & 11 \\
\hline $\mathrm{J} 2207+1652$ & 215 & 50 & & & 248 & 12 & 225 & 6 & 11 \\
\hline $\mathrm{J} 2320+0513$ & 623 & 62 & & & 656 & 10 & 721 & 6 & $19(4.5 \mathrm{GHz})$ \\
\hline
\end{tabular}

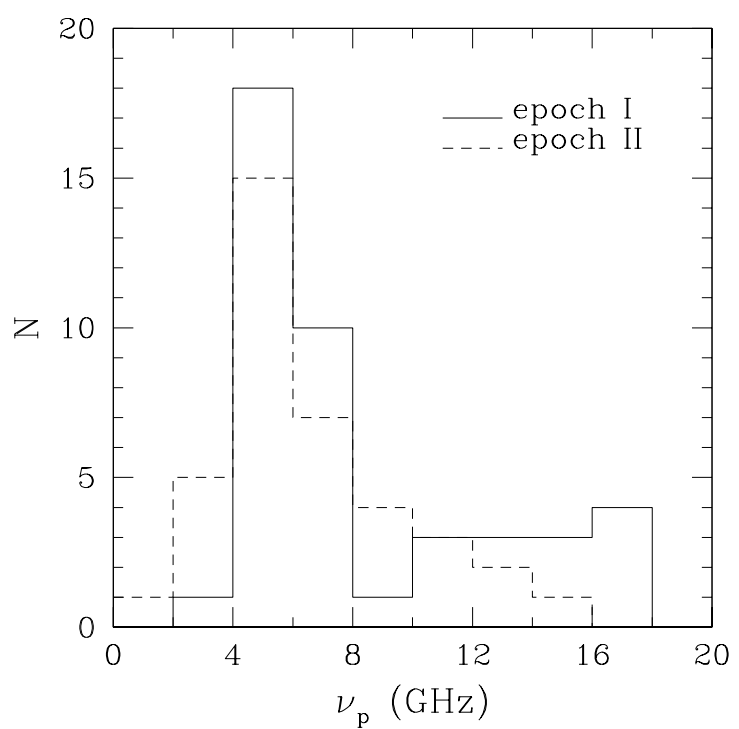

Fig. 3. Histogram of observed turnover frequencies for the HFP sample at the two epochs.

analyzing high resolution VLBA observations of J0428 + 3259 to study its pc scale morphology.

\section{Analysis of the sample}

The repeated high sensitivity simultaneous multifrequency observations separated by 3 to 4 years allow us to investigate the variability properties of our candidate HFPs. This analysis may also help discriminating between truly young sources and flaring blazar components.

The convex form of the spectrum is the first property that should be preserved in the evolution of young sources. This does not happen for 7 sources (16\% of the sample), all identified with quasars ( $25 \%$ of such objects), whose second epoch spectrum turns out to be flat. Such sources are labelled as "flat" in Col. 9 of Table 2, and are then classified as blazars.

According to the physical model for the evolution of young radio sources by Begelman (1996), a newly generated powerful jet injects energy into the ambient medium inflating a cocoon consisting of shocked jet material and shocked ambient matter. If the density of the surrounding medium scales with radius, $r$, as $\rho \propto r^{-n}$, the linear size, $L S$, of the cocoon increases with the source age, $t$, as $L S \propto t^{\epsilon}$, with $\epsilon=3 /(5-n)$. The best determined relationship for GPS/CSS sources relates the radio turnover frequency to the projected linear size $v_{\mathrm{p}} \propto(L S)^{-\delta}$ (O'Dea \& Baum 1997). It follows that $v_{\mathrm{p}}$ decreases with time as $v_{\mathrm{p}} \propto t^{-\lambda}$, with $\lambda=\delta \cdot \epsilon$. The expected decrease of $v_{\mathrm{p}}$ in a time interval $\Delta t$ in the source frame (corresponding to $\Delta T=\Delta t(1+z)$ in the observer frame) is then:

$\frac{\Delta v_{\mathrm{p}}}{v_{\mathrm{p}}}=-\lambda \frac{\Delta t}{t}$.

The slope of the observed correlation for CSS/GPS sources is $\delta \simeq 0.65$ but O'Dea \& Baum (1997) show that the evolution of $v_{\mathrm{p}}$ with LS may be steeper than the statistical relation. Assuming Begelman's model, they found $\delta \simeq 1.2$ for $n=2$.

The typical uncertainties associated with our estimates of $\Delta v_{\mathrm{p}}$ are at the several percent level. For $n$ ranging from 0 (uniform density) to 2 (isothermal distribution) and $\Delta T=$ 3-4 yr, a clear measurement of the peak frequency decrease due to the source expansion could be achieved for source ages well below 100 yr. Taking into account that our sources were selected from the GB87 catalogue, based on observations carried out in 1986-1987, and therefore must have been older than 15-16 yr when our second set of observations were done, we conclude that sources with $\Delta v_{\mathrm{p}} /\left[v_{\mathrm{p}} \Delta t(\mathrm{yr})\right]>0.05$ (last column of Table 2) are unlikely to be HFPs (because too young ages would be implied). We propose to classify them as blazars, whose observed evolution timescale are decreased by the Doppler factor. Note that $\Delta v_{\mathrm{p}} /\left[v_{\mathrm{p}} \Delta t(\mathrm{yr})\right]$ can be computed 

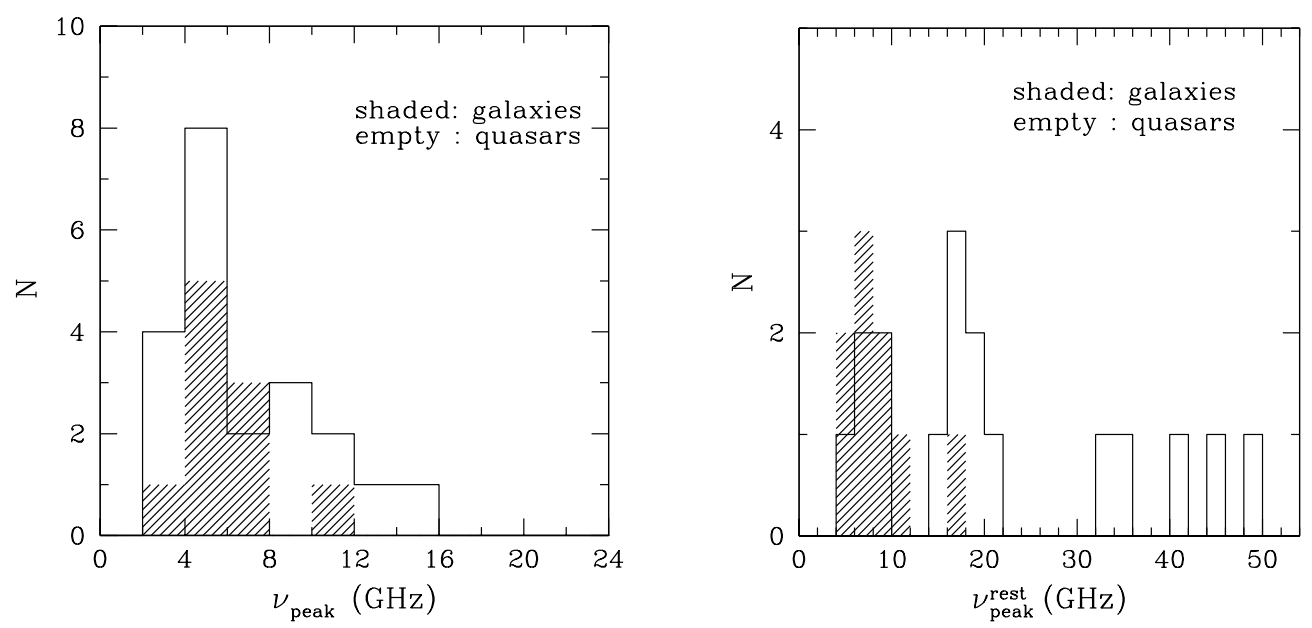

Fig. 4. Observed (left) and rest frame (right) turnover frequency distributions for galaxies and quasars.
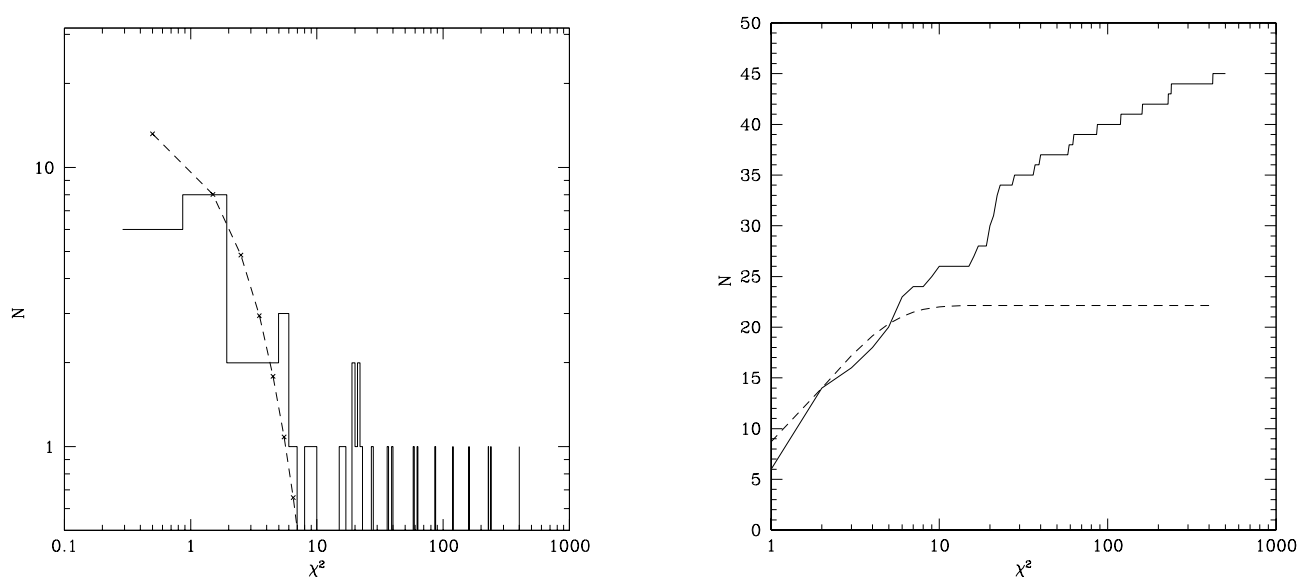

Fig. 5. Differential (left) and cumulative (right) distribution of $V$, compared with the corresponding $\chi^{2}$ distributions for 2 degrees of freedom (dashed lines). The bins of the differential distribution correspond to $\Delta \chi^{2}=1$.

(and is reported in Table 2) only for sources with measured redshift and measured peak frequencies at both epochs.

In principle, an estimate of source ages could be derived from Eq. (3). In practice, however, given the large uncertainties on $\Delta v_{\mathrm{p}}$ and the additional uncertainties on $\lambda$ (due to the poor knowledge of the parameter $n$ characterizing the density profile of the medium), only rather uninteresting lower limits of a few tens of years can be set. On the other hand, interesting constraints would be provided by more accurate multifrequency measurements with a 2 or 3 times longer time-lag.

Although the Kolmogorov-Smirnov (KS) test does not detect any significant difference in the distribution of turnover frequencies at the two epochs, it is clear from the last column of Table 2 that most sources have a smaller $v_{\mathrm{p}}$ at the second epoch, consistent with an evolution towards lower optical depths.

Flux density variability also helps distinguishing between expanding young objects and aged beamed objects (blazars) whose radio emission is dominated by a single knot in the jet. While HFP/GPS sources are thought to expand at mildly relativistic velocities, the large Doppler factors characterizing blazar jets boost the variability amplitudes and decrease the corresponding observed timescales. We have analyzed the variability of our sources in terms of the quantity

$V=\frac{1}{m} \sum_{i=1}^{m} \frac{\left(S_{\mathrm{I}}(i)-S_{\mathrm{II}}(i)\right)^{2}}{\sigma_{i}^{2}}$,

where $S_{\mathrm{I}}(i)$ and $S_{\mathrm{II}}(i)$ are the flux densities at the $i$-th frequency, measured in the first and second epoch, respectively, $\sigma_{i}$ is the error on $S_{\mathrm{I}}(i)-S_{\mathrm{II}}(i)$, and $m$ is the number of the sampled frequencies.

We have noted that the second epoch flux densities at $1.4 \mathrm{GHz}$ are systematically higher by about $10 \%$ than the first epoch ones, while the flux densities at $1.7 \mathrm{GHz}$ at the two epochs appear to match. This may be related to an unidentified problem during the $1.4 \mathrm{GHz}$ observation of the primary calibrator 3C 286. We have therefore chosen to exclude the 1.4 GHz measurements, so that in the above equation $m=7$.

For HFP sources, $V$ depends essentially on only two free parameters, $S_{\mathrm{p}}$ and $v_{\mathrm{p}}$ (since the spectral slopes are not expected to vary), and should therefore have a $\chi^{2}$ distribution with 2 degrees of freedom. Figure 5 shows that indeed sources with low values of $V$ obey such distribution. Since the probability that a source with $V>9$ is extracted from such distribution 
is $<0.01$, we conclude that sources with $V>9$ are very likely blazars. On the other hand, blazars may well have small values of $V$, so that there is no guarantee that sources with $V<9$ are all truly young. We caution however that these conclusions rely to some extent on our estimates of errors, dominated by calibration uncertainties: any significant over- or under-estimate of errors would impair the match of the distribution of $V$ with the $\chi^{2}$ distribution with 2 degrees of freedom.

As shown in Table 2, all 10 galaxies, including the Seyfert 1 , have $V<9$, while most quasars (17 out of 28 , including the 7 with flat second-epoch spectra) have $V>9$; of the 7 unidentified sources, 4 have $V<9$. Thus, if we confine ourselves to sources with $V<9$, we have an almost equal number of galaxies and quasars, similarly to what is found for GPS samples. The quasars with $V<9$ have higher median redshift $\left(z_{\text {median }} \simeq 2.4\right)$ than those with $V>9$, whose median redshift $\left(z_{\text {median }} \simeq 1.7\right)$ is closer to that of flat spectrum radio quasars in the Parkes quarter-Jy sample $\left(z_{\text {median }} \simeq 1.4\right)$.

An alternative approach may be to consider as blazar candidates all sources with variability significant at more than $3 \sigma$. In this case the boundary between candidate HFP and likely blazars has to be set at $V=3$ (as appropriate for a $\chi^{2}$ distribution with 1 degree of freedom) rather than at $V=9$. If so, 9 more sources (3 galaxies, including the type 1 Seyfert, 5 quasars and 1 unidentified source) add to the list of candidate blazars.

Another interesting issue is the relationship between peak frequencies and peak luminosities, $L_{\mathrm{p}}$. Opposite trends are predicted by current models for GPS sources: while Begelman's (1996) model implies a decrease of $L_{\mathrm{p}}$ with decreasing $v_{\mathrm{p}}$, Snellen et al. (2000) predict an increase during the HFP/GPS phase of the source evolution. In both cases, however, the dependence of $L_{\mathrm{p}}$ on $v_{\mathrm{p}}$ is weak, and therefore easily swamped by variances in both quantities. In the case of relativistic beaming with Doppler factor $\delta$, the observed peak luminosities scale as $\delta^{3}$, while the observed peak frequencies scale as $\delta$, so that sources with equal intrinsic $L_{\mathrm{p}}$ and $v_{\mathrm{p}}$, but different $\delta$ 's are observed to have $L_{\mathrm{p}} \propto v_{\mathrm{p}}^{3}$; this relation is much steeper than the $L_{\mathrm{p}}-v_{\mathrm{p}}$ relation predicted by both Begelman's and Snellen's models.

We have investigated this relationship for all objects (see Fig. 6), using first epoch data, when all sources but two had a well defined spectral peak. Although a clear correlation seems to be present, we must beware of the effect of the redshift distribution: as an example, the solid line in Fig. 6 shows the distribution in the $L_{\mathrm{p}}-v_{\mathrm{p}}$ plane of sources with equal observed $S_{\mathrm{p}}=300 \mathrm{mJy}$ and $v_{\mathrm{p}}=5 \mathrm{GHz}$ at different redshifts. Clearly the shape of the correlation is not far from that induced by the effect of redshift. Nevertheless, Kendall's partial correlation coefficient indicates a statistically significant positive correlation between $L_{\mathrm{p}}$ and $v_{\mathrm{p}}$ after the influence of redshift has been eliminated. The probability of no correlation is $0.44 \%$ if we consider only stellar objects with $V>9$ (which are probably blazars; we have 12 such objects with redshift, but for one of them the peak frequency is undefined). For stellar objects with $V \leq 9$ the probability of no correlation increases to $6 \%$ so that the correlation is only marginally significant. For galaxies there is no

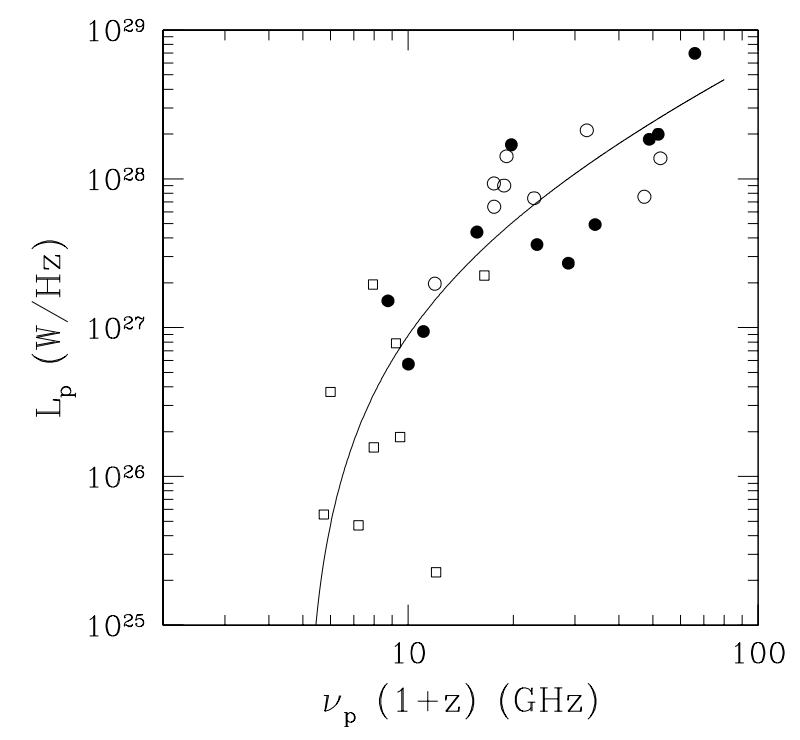

Fig. 6. Peak luminosity versus peak frequency for all objects with measured redshift. Circles: quasars, squares: galaxies. Open symbols refer to sources with $V \leq 9$, filled symbols to sources with $V>9$. The solid line shows the distribution in the $L_{\mathrm{p}}-v_{\mathrm{p}}$ plane of sources with equal observed $S_{\mathrm{p}}=300 \mathrm{mJy}$ and $v_{\mathrm{p}}=5 \mathrm{GHz}$ at different redshifts.

indication of a significant correlation (probability of no correlation $38 \%$ ).

Models for GPS/HFP sources also predict a correlation between peak luminosity and linear size, whose slope is related to the slope $n$ of the density profile of the surrounding medium. Unfortunately, our VLA observations provide only upper limits to the source sizes, since sources are unresolved. A search of databases available in the VLBA Web site has yielded maps at various frequencies for 15 out of the 18 sources with $V \leq 9$ and known redshift. Of these, 8 were resolved and for them we estimated the angular size. For the remaining 10 we adopted upper limits corresponding to the beam size. One source has not been included in the analysis because it has not a defined spectral peak at the first epoch of VLA observations. These data show no indication of a correlation between the linear size and the peak luminosity (probability of no correlation $\simeq 0.7$ ) and do not confirm, for our sample, the correlation between peak flux density and angular size (probability of no correlation $\simeq 0.6$ ) reported by Snellen et al. (2000) for their GPS sample.

\section{Discussion and conclusions}

The spectral selection adopted by Dallacasa et al. (2000) is independent of optical identification and redshift. As a result, both galaxies and star-like objects ("quasars") are present in the original sample of bright HFP candidates. Quasars are almost three times more numerous than galaxies, consistent with the previously noted decrease of the galaxy to quasar ratio in GPS samples selected at increasing turnover frequency (Fanti et al. 1990; O'Dea 1998; Stanghellini et al. 1998, 2003).

However, our new multifrequency observations, with a time lag of 3 to 4 years, indicate that the sample of candidate HFP quasars is likely to be strongly contaminated by beamed 
objects, with variability properties consistent with those of blazars.

Seven out of the 28 star-like sources $(25 \%)$ no longer have a peaked spectrum, and 5 of them have extended emission. Such sources are therefore classified as blazars, caught by Dallacasa et al. (2000) during a flaring phase of a strongly self-absorbed component.

Seven additional star-like sources show a variation of the peak frequency, significant at a $>3 \sigma$ level, larger than expected if HFP/GPS sources expand at mildly relativistic velocities $\left(\Delta v_{\mathrm{p}} /\left[v_{\mathrm{p}} \Delta t(\mathrm{yr})\right]>0.05\right)$, suggesting the presence of relativistic beaming effects. The only galaxy $(1407+2827)$ with a peak frequency variation significant at $>3 \sigma$ level has $\Delta v_{\mathrm{p}} /\left[v_{\mathrm{p}} \Delta t(\mathrm{yr})\right]=$ 0.017 .

Another criterion to discriminate between truly young sources and blazar is the variability index $V$ (Eq. (4)). The distribution of $V$ for the less variable sources is close to the $\chi^{2}$ distribution with two degrees of freedom, as may be expected since variations of spectra of young sources are controlled by two parameters, $S_{\mathrm{p}}$ and $v_{\mathrm{p}}$. If so, sources with $V>9$ have a probability $<0.01$ of being extracted from such a distribution and are therefore likely blazars. We have 20 sources with $V>$ 9, 17 of which are star-like (including the 7 with flat spectrum at the second epoch and 4 of the 7 with $\left.\Delta v_{\mathrm{p}} /\left[v_{\mathrm{p}} \Delta t(\mathrm{yr})\right]>0.05\right)$ and 3 unidentified sources. The median rms variations for these sources are $\simeq 30 \%$ (27\% for quasars only), close to those found for blazars ( $\simeq 32 \%$; Ciaramella et al. 2004).

The variability timescales in the frequency range $5-15 \mathrm{GHz}$ of bright blazars, derived from their structure function, are $\simeq 2 \mathrm{yr}$ in the source frame (Hughes et al. 1992; Ciaramella et al. 2004). To convert the time-lag between the two sets of observations into the mean time interval in the source frame, we need to divide it by $\left(1+z_{\text {mean }}\right)$. Only 11 of the 17 starlike sources with $V>9$ have measured redshift, with a median value of 1.7. We may thus expect that $\sim 50 \%$ of blazars flaring at the moment of the first set of observations have recovered their baseline flat spectrum by the epoch when they where re-observed. For comparison, 7 out of 17 (i.e. $\simeq 41 \%$ ) were indeed found to have a flat spectrum in the second observing run.

There is evidence of a positive correlation between $V$ and $v_{\mathrm{p}}$ (probability of no correlation $\sim 0.01$ ), indicating that the fraction of blazars is higher among objects with higher peak frequency. This is not surprising because young objects become increasingly rare since their lifetimes decrease with increasing $v_{\mathrm{p}}$.

We have found a statistically significant positive correlation between the peak luminosity and rest-frame peak frequency of quasars, after having removed the effect of the redshift distribution (Kendall's partial correlation test). A relationship among these two quantities is expected both in the case of GPS/HFP sources and of blazars, but with a much steeper slope for the latter sources. While previous studies enlightened a $S_{\mathrm{p}}-v_{\mathrm{p}}$ correlation for a few individual blazars (e.g., Stevens et al. 1996) our analysis indicates that it holds for flaring blazars as a class.

There are thus various pieces of evidence, albeit circumstantial, that most quasar HFP candidates are actually objects where the effects of beaming are relevant (blazar like) even if this does not necessarily require that we are seeing evolved/old objects.

While sources with $V>9$ are likely blazars there is no guarantee that those with $V<9$ are truly young sources. Some of them may really be blazars in a relatively quiescent phase. The 3 such objects with $\Delta v_{\mathrm{p}} /\left[v_{\mathrm{p}} \Delta t(\mathrm{yr})\right]>0.05$ may belong to this category.

As for galaxies, all $10 \mathrm{HFP}$ candidates have $V<9$. Their peak luminosities and rest-frame peak frequencies do not show evidence of a correlation, not surprisingly given the smaller range spanned by both quantities (compared to the case of quasars) and the weaker dependence of $L_{\mathrm{p}}$ on $v_{\mathrm{p}}$ expected for GPS/HFP sources, easily swamped by intrinsic dispersions of both quantities.

Two galaxies show extended emission compatible with recurrent activity. According to Begelman's model the synchrotron emission decreases with source age $t$ as $L \propto t^{-\eta}$ with $\eta=(n+4) /[4(5-n)]$. In the case of recurrent activity, the HFP to extended emission age ratio can then be estimated as $t_{\mathrm{HFP}} / t_{\mathrm{ext}}=\left(L_{\mathrm{ext}} / L_{\mathrm{HFP}}\right)^{1 / \eta}$, where $L_{\mathrm{ext}}$ and $L_{\mathrm{HFP}}$ are the total emitted radio luminosities, that can be taken as proportional to the emitted flux densities at $1.4 \mathrm{GHz}$. In the case of the HFP component, the latter can be estimated extrapolating to $1.4 \mathrm{GHz}$ the peak flux density. The age ratio derived from Begelman's model depends on the slope $n$ of the density profile of the surrounding medium. Adopting $n=2$ we get, for $\mathrm{J} 0111+3906, t_{\mathrm{ext}} / t_{\mathrm{HFP}} \simeq 9 \times 10^{4}$, and for $\mathrm{J} 0428+3259$ $t_{\mathrm{ext}} / t_{\mathrm{HFP}} \simeq 2 \times 10^{4}$, consistent with the intermittency timescales suggested by Reynolds \& Begelman (1997). Although any conclusion at this stage is premature, this illustrates the potential of deeper low frequency observations to shed light on the nature of radio activity.

In conclusion, while the variability properties of candidate HFP galaxies may be consistent with expectations for truly young, possibly recurrent, sources, those of most quasars are consistent with those of blazars. If we set the boundary between HFP candidates and blazars at $V=3$ (see Sect. 5), 3 galaxies would host a blazar nucleus, and 22 out of the 28 starlike sources and 4 out of the 7 unidentified sources would be blazar candidates. The median variability amplitude of sources with $V>3$ is $\simeq 22 \%$, slightly lower than, but still not far from that found for blazar samples. Milli-arcsec morphology at different frequencies, polarization measurements and possibly the study of the spectral index distributions in the optically thin regime can help distinguishing between flaring blazars and truly young objects.

High resolution VLBA observations at two frequencies in the optically thin part of the spectrum (between 8.4 and $43.2 \mathrm{GHz}$ ) of 50 objects of the original sample have already been acquired. The analysis of the data is in progress.

Acknowledgements. We thank the referee, Prof. Roberto Fanti, for useful comments that helped improving substantially the manuscript. The VLA is operated by the US National Radio Astronomy Observatory which is a facility of the National Science Foundation operated under a cooperative agreement by Associated Universities, Inc. This work has made use of the NASA/IPAC Extragalactic Database NED which is operated by the JPL, California Institute of Technology, under contract with the National Aeronautics and Space 
Administration. The authors acknowledge financial support from the Italian MIUR under grant COFIN-2002-02-8118.

\section{References}

Baum, S. A., O’Dea, C. P., de Bruyn, A. G., et al. 1990, A\&A, 232, 19

Begelman, M. C. 1996, in Proc. Cygnus A - Study of radio Galaxy, ed. C. L. Carilli, \& D. E. Harris (Cambridge: CUP), 209

Bicknell, G., Dopita, M. A., \& O’Dea, C. P. 1997, ApJ, 485, 112

Browne, I. W. A., Wilkinson, P. N., Patnaik, A. R., et al. 1998, MNRAS, 293, 257

Ciaramella, A., Bongardo, C., Aller, H. D., et al. 2004, A\&A, 419, 485

Condon, J. J., Cotton, W. D., Greisen, E. W., et al. 1998, AJ, 115, 1693

Dallacasa, D. 2003, PASA, 20, 79

Dallacasa, D., Stanghellini, C., Centonza, M., et al. 2000, A\&A, 363, 887

Dallacasa, D., Falomo, R., \& Stanghellini, C. 2002a, A\&A, 382, 53

Dallacasa, D., Fanti, C., Giacintucci, S., et al. 2002b, A\&A, 389, 126

Dallacasa, D., Tinti, S., Fanti, C., et al. 2002c, A\&A, 389, 115

Fanti, C., \& Fanti, R. 2002, in Issues in Unification of Active Galactic Nuclei, ed. R. Maiolino, A. Marconi, \& N. Nagar (San Francisco: ASP), ASP Conf. Ser., 258, 261

Fanti, R., Fanti, C., Schilizzi, R. T., et al. 1990, A\&A, 231, 333

Fanti, C., Pozzi, F., Fanti, R., et al. 2000, A\&A, 358, 499

Gregory, P. C., Scott, W. K., Douglas, K., et al. 1996, ApJS, 103, 427

Hughes, P. A., Aller, H. D., \& Aller, M. F. 1992, ApJ, 396, 469

Impey, C. D., \& Neugebauer, G. 1988, AJ, 95, 307
Jackson, C. A., Wall, J. V., Shaver, P. A., et al. 2002, A\&A, 386, 97

Murgia, M., Fanti, C., Fanti, R., et al. 1999, A\&A, 345, 769

O'Dea, C. P., \& Baum, S. A. 1997, AJ, 113, 148

O'Dea, C. P. 1998, PASP, 110, 493

Owsianik, I., \& Conway, J. E. 1998, A\&A, 337, 69

Owsianik, I., Conway, J. E., \& Polatidis, A. G. 1998, A\&A, 336, L37

Patnaik, A. R., Browne, I. W. A., Wilkinson, P. N., et al. 1992, MNRAS, 254, 655

Reynolds, C. S., \& Begelman, M. C. 1997, ApJ, 487, L135

Snellen, I. A. G., Bremer, M. N., \& Schilizzi, R. T. 1996, MNRAS, 279, 1294

Snellen, I. A. G. 1997, Ph.D. Thesis, University of Leiden

Snellen, I. A. G., Schilizzi, R. T., \& de Bruyn, A. G. 1998, A\&AS, 131,435

Snellen, I. A. G., Schilizzi, R. T., Miley, G. K., et al. 2000, MNRAS, 319,445

Stanghellini, C., Baum, S. A., O'Dea, C. P., et al. 1990, A\&A, 233, 379

Stanghellini, C., O’Dea, C. P., Dallacasa, D., et al. 1998, A\&AS, 131, 303

Stanghellini, C. 2003, PASA, 20, 118

Stevens, J. A., Litchfield, S. J., Robson, E. I., et al. 1996, ApJ, 466, 158

Taylor, G. B., Readhead, A. C. S., \& Pearson, T. J. 1996, ApJ, 463, 95 van Breugel, W., Heckman, T., \& Miley, G. 1984, ApJ, 276, 79

Wilkinson, P. N., Browne, I. W. A., Patnaik, A. R., et al. 1998, MNRAS, 300, 790 
S. Tinti et al.: High Frequency Peakers: Young radio sources or flaring blazars?, Online Material p 1

\section{Online Material}




\section{Appendix A: Notes on the individual sources}

J0111+3906: Well known galaxy at $z=0.668$. We have detected extended emission on kpc scale (see Fig. A.1), already discovered and imaged by Baum et al. (1990), Taylor et al. (1996), Stanghellini (2003), at different resolutions. The extended emission is in contrast with a recent origin for the radio activity in this source but can be explained if the radio source is recurrent (Baum et al. 1990).

J0217+0144: Quasar at $z=1.715$. The source is slightly resolved in the NW-SE direction in the L band. At higher resolution, in the $C$ and $\mathrm{X}$ bands, two opposite regions of emission with respect to the central compact component are detected (see Fig. A.2).

J0329+3510: Stellar object. We see structure on arcsecond scale (lobes-hotspots). The extended emission is still detected, even if very weak, in the $\mathrm{C}$ band (see Fig. A.3); a jet like feature is emerging from the core towards the brightest lobe.

J0428+3259: Galaxy with estimated redshift $z=0.3$ (Dallacasa et al. 2002), based on the Hubble diagram in Snellen et al. (1996). The source is slightly resolved along the NW-SE axis at both $1.465 \mathrm{GHz}$ and $1.665 \mathrm{GHz}$ (Fig. A.4), but no resolved emission is found at $5 \mathrm{GHz}$, due to sensitivity limitations.

J0519+0848: Optically unidentified. It is slightly resolved with a hint of weak emission in the NW direction (see Fig. A.5).

J1457+0749: Optically unidentified. It is slightly resolved in the NW direction (see Fig. A.6).

J1603+1105: Optically unidentified. The NE component can be either associated with the central object or can be an unrelated source (see Fig. A.7); according to Snellen (1998) there is a $6 \%$ chance of finding an unrelated NVSS radio source with a flux density of $>5 \mathrm{mJy}$ within a radius of $100 \mathrm{arcsec}$ from the target source.

J1800+3848: Quasar at $z=2.092$. At 1.465 and $1.665 \mathrm{GHz}$ the source appears slightly resolved but the estimated flux density of the extended emission is of a few mJy only (see Fig. A.8).

J1811+1704: stellar object. A very weak component at NW is clearly visible both at $1.465 \mathrm{GHz}$ and $1.665 \mathrm{GHz}$ (see Fig. A.8). J2024+1718: Stellar object at $z=1.050$. The emission is extending from the core to the North for $11 \mathrm{arcsec}$ (see Fig. A.9). J2101+0341: Quasar at $z=1.013$. This source has a very complex structure. Two components are aligned with the NS axis for a total angular size of 14 arcsec. Furthermore there is a diffuse low-brightness emission in the NE direction with angular size of 22 arcsec (see Fig. A.9).

J2123+0535: Quasar at $z=1.878$. There is a very weak emission toward the South of the strong compact component (see Fig. A.10).

J2207+1652: Stellar object. On arcsec scales it shows two components, the southern one being weaker and smaller. This component is still clearly visible in the $\mathrm{X}$ band (see Fig. A.11).

J2320+0513: Quasar at $z=0.622$. Extended emission is revealed in both the $L$ and the $\mathrm{C}$ band. The maximum angular size in the NW-SE direction is of 19 arcsec. In the $\mathrm{C}$ band the extended emission has two irregular components on both sides of the unresolved nucleus, the southern one being weaker than the northern one (see Fig. A.12). 
S. Tinti et al.: High Frequency Peakers: Young radio sources or flaring blazars?, Online Material p 3
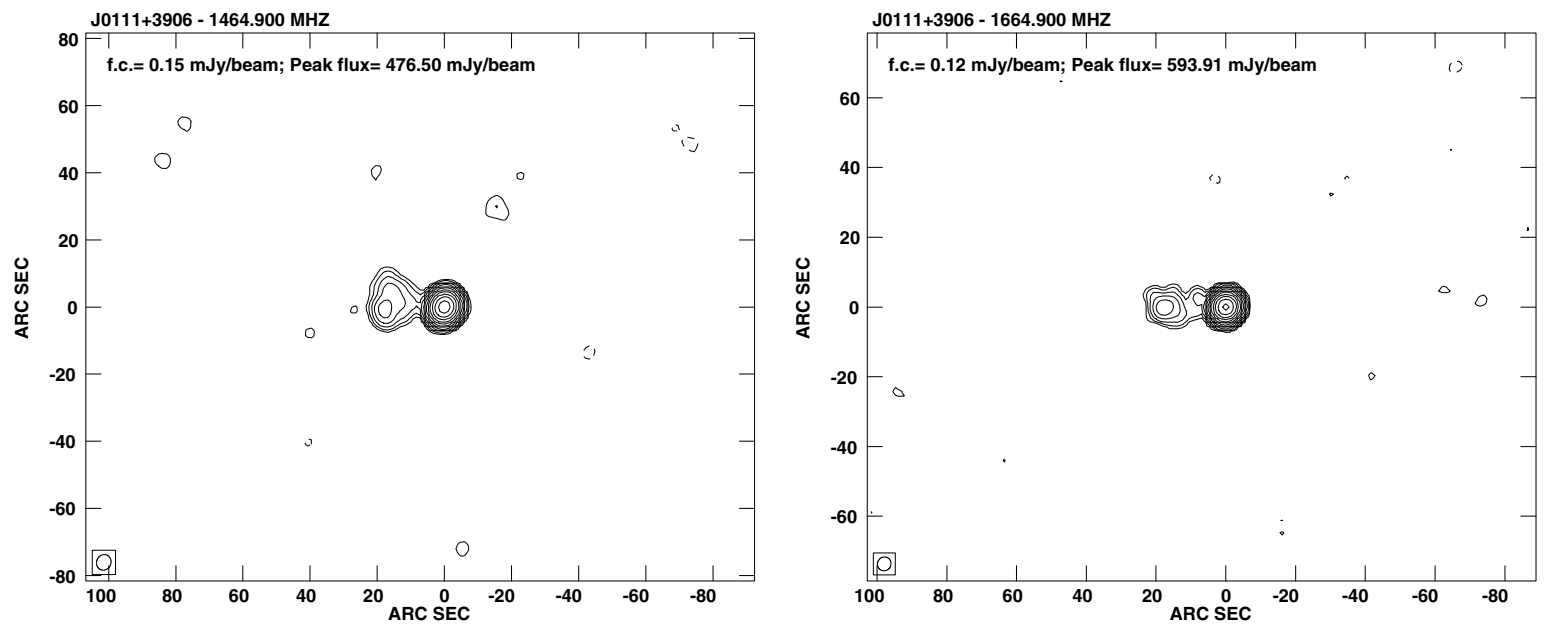

Fig. A.1. J0111+3906; the restoring beam is in the Bottom Left Corner of each figure, the conturn levels are $\pm 1,2,4,8,16,32$, 64, 128, 256, $512,1024,2048,4096$ times the first conturn (f.c.) corresponding to 3 times the rms noise of the image.
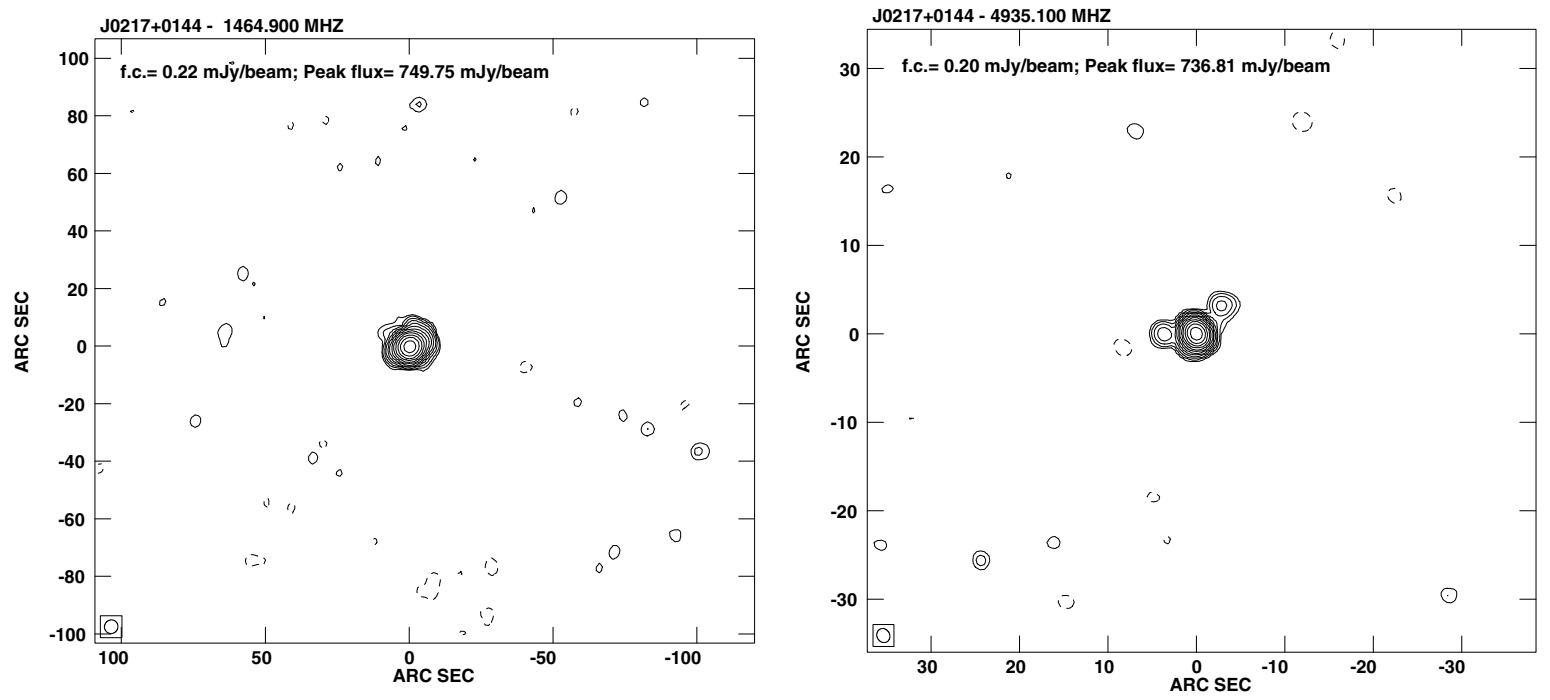

Fig. A.2. J0217+0144.
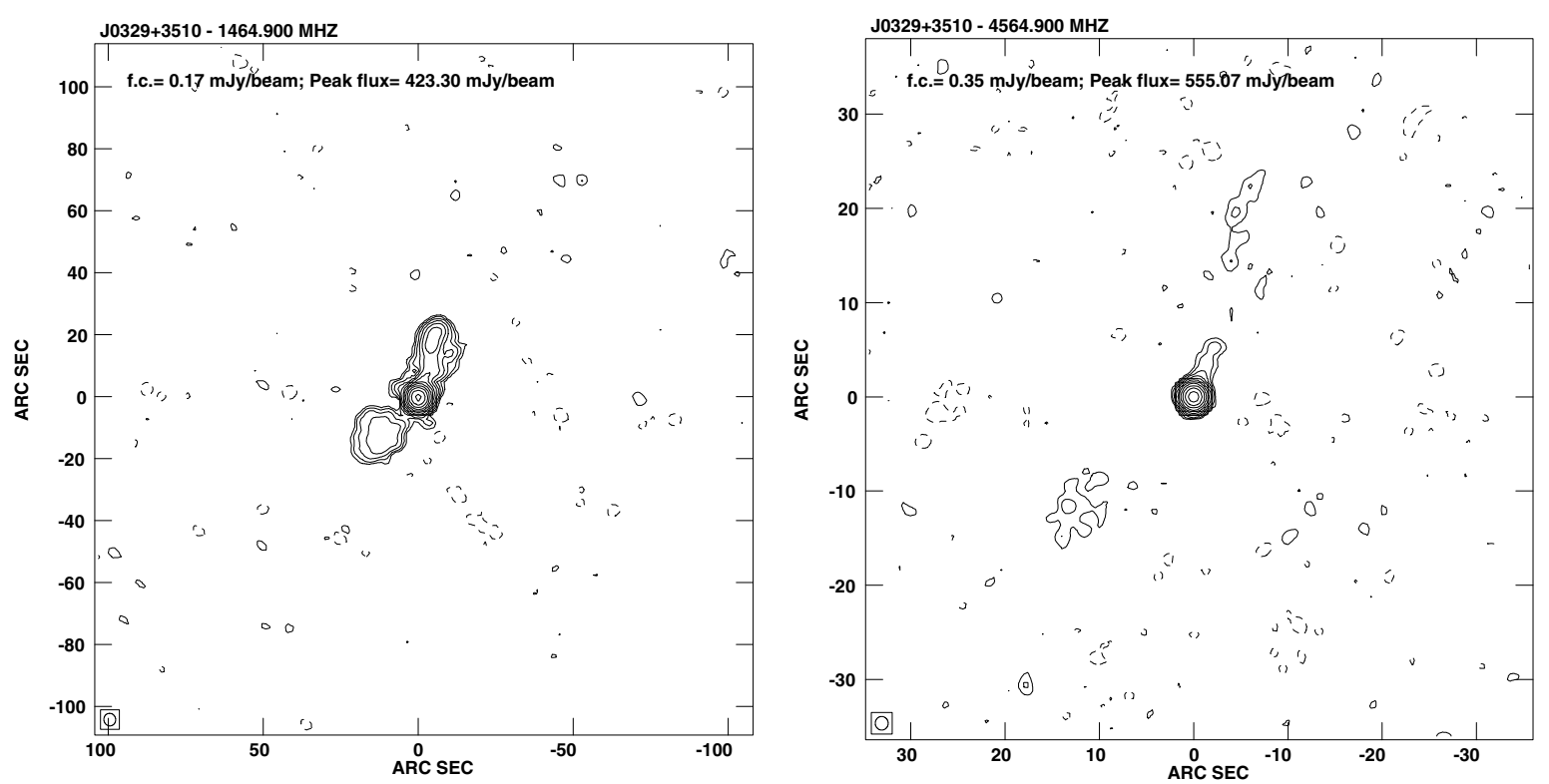

Fig. A.3. J0329+3510. 
S. Tinti et al.: High Frequency Peakers: Young radio sources or flaring blazars?, Online Material p 4
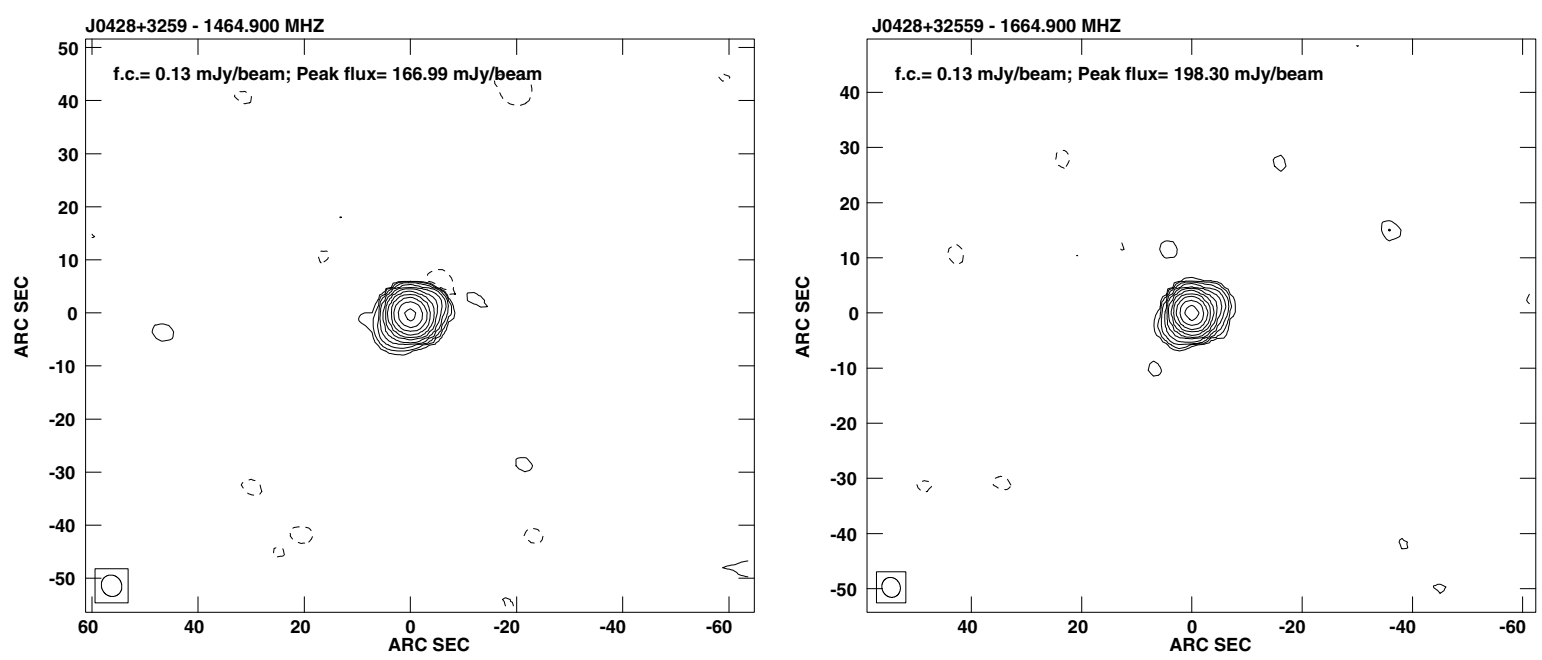

Fig. A.4. J0428+3259.
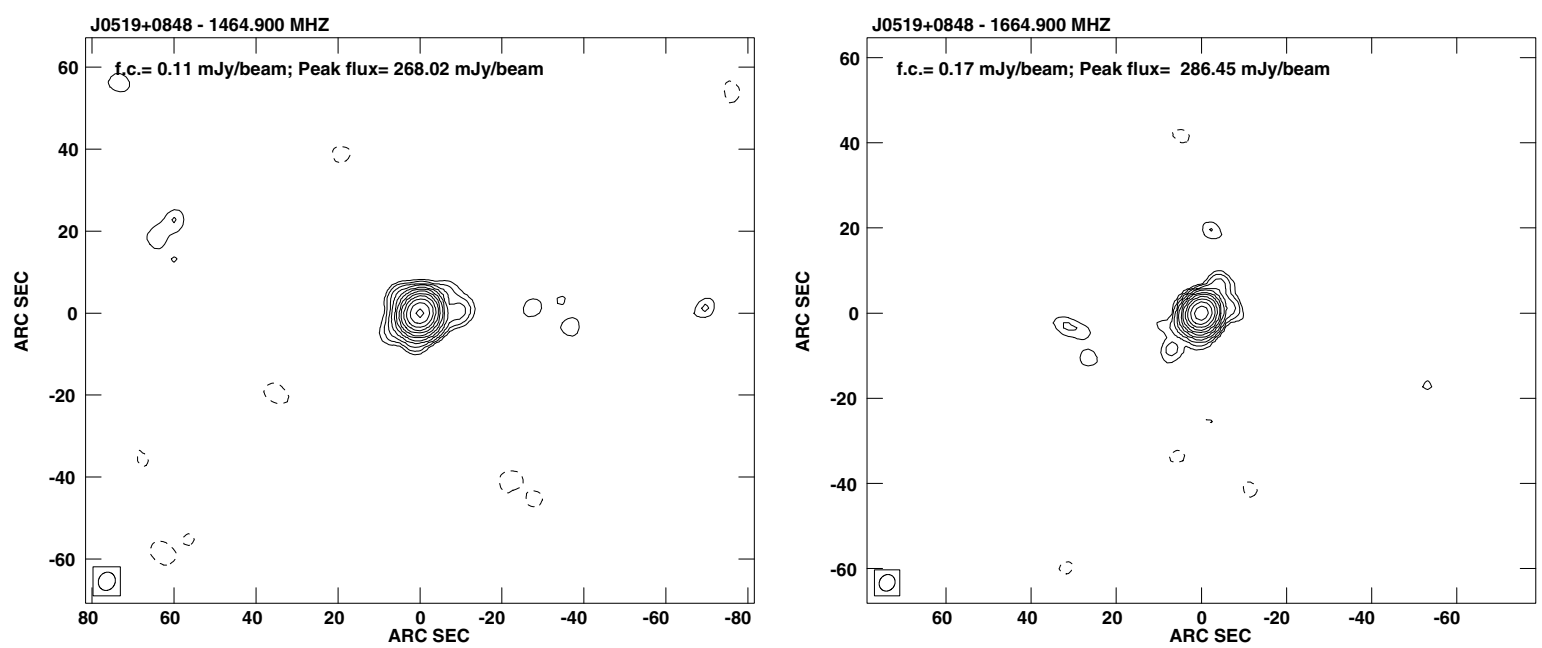

Fig. A.5. J0519+0848.
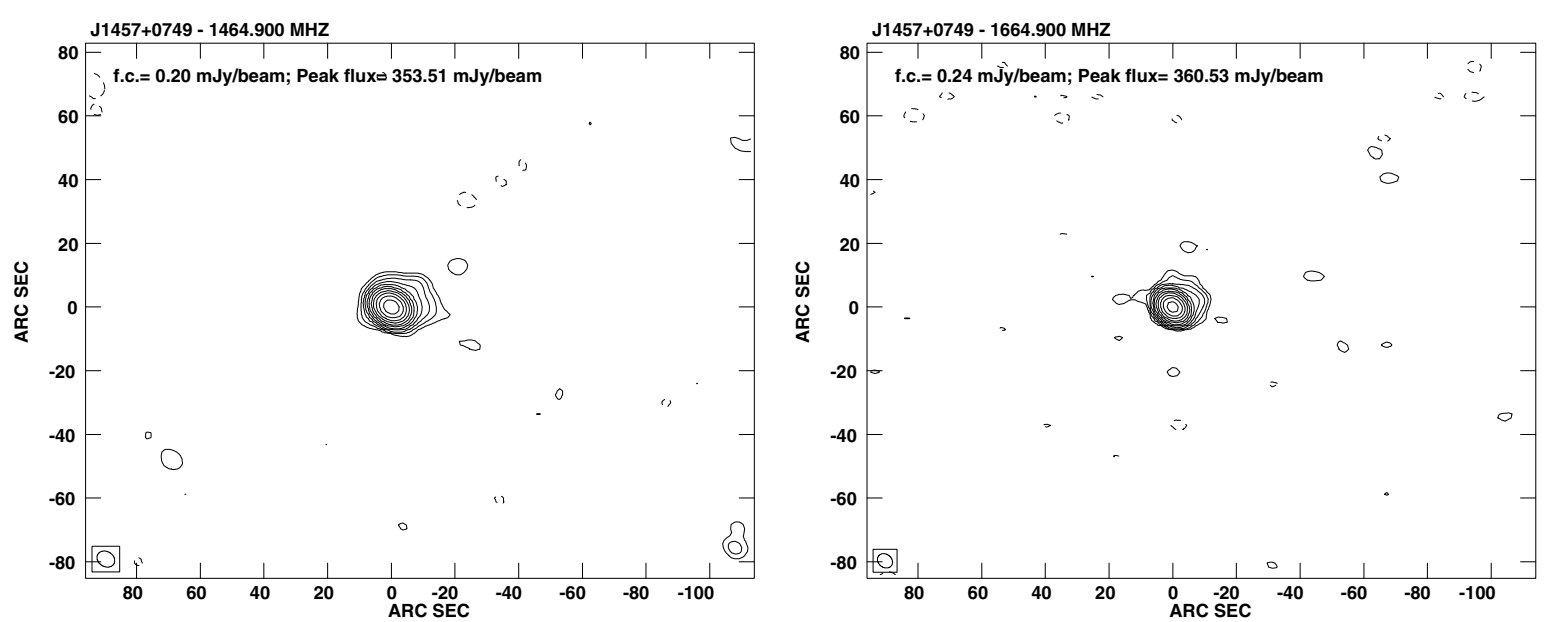

Fig. A.6. J1457+0749. 
S. Tinti et al.: High Frequency Peakers: Young radio sources or flaring blazars?, Online Material p 5
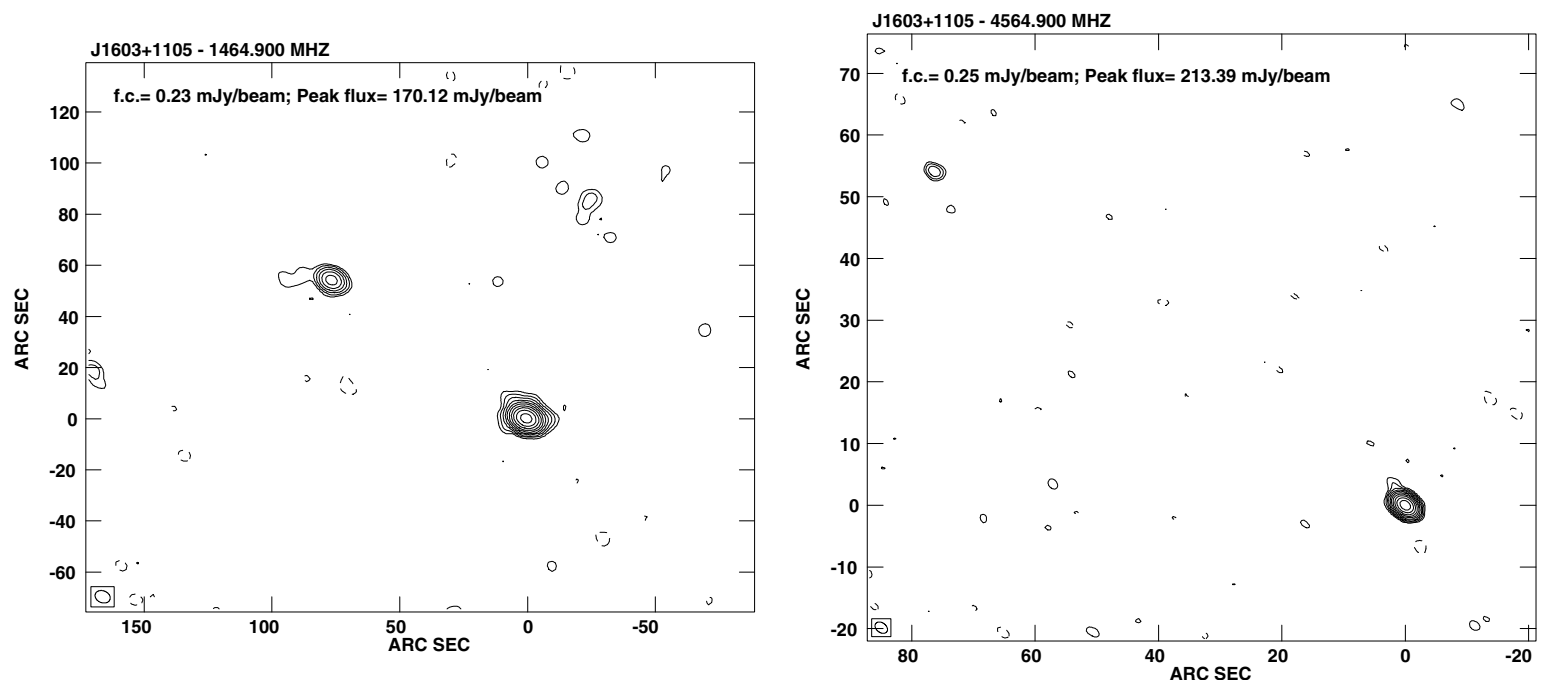

Fig. A.7. J1603+1105.
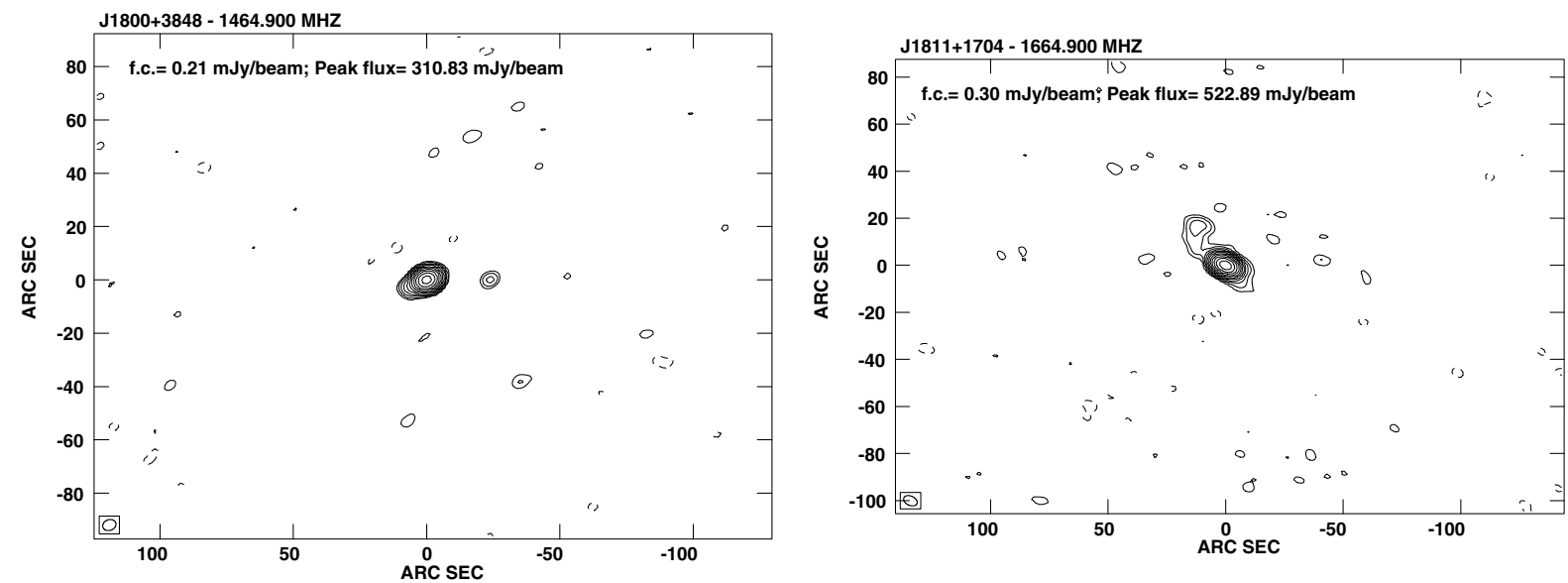

Fig. A.8. J1800+3848 (left) at $1.465 \mathrm{GHz}$ and $\mathrm{J} 1811+1704$ (right) at $1.665 \mathrm{GHz}$.
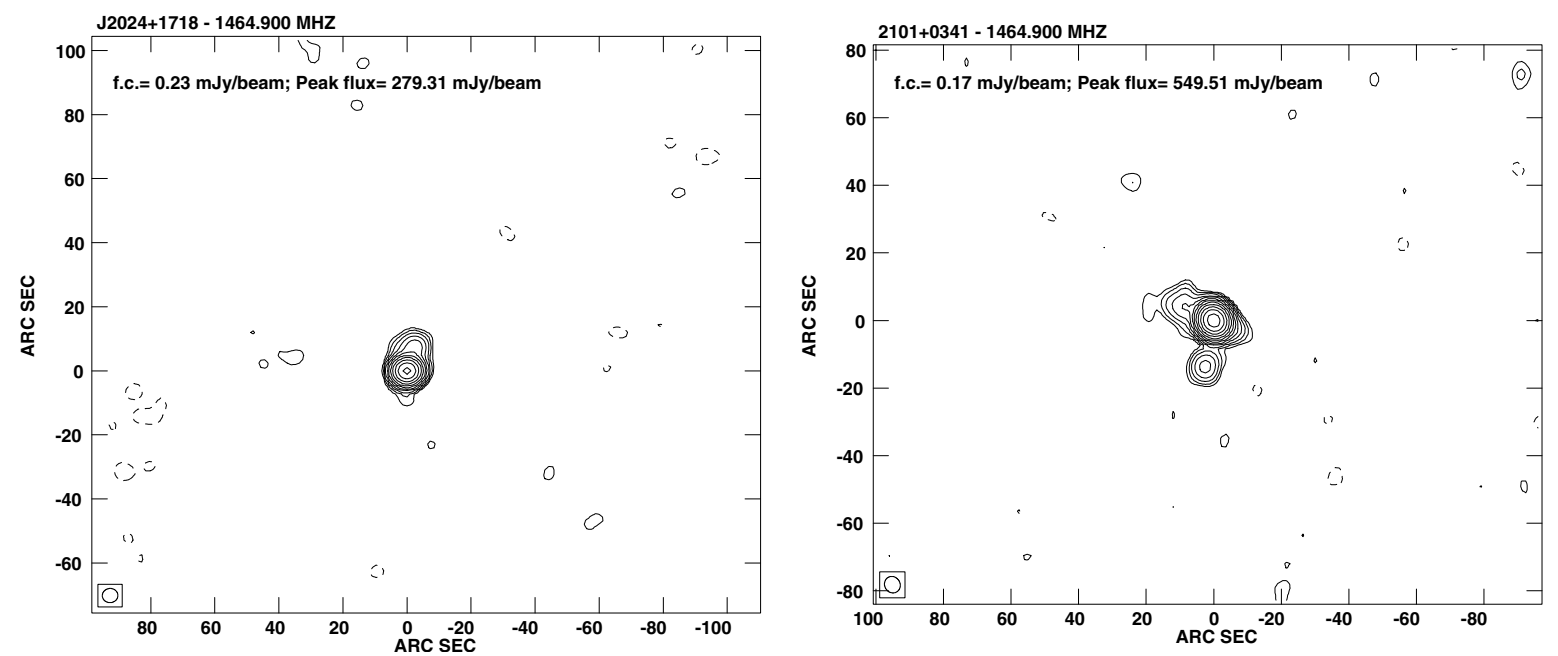

Fig. A.9. J2024+1718 (left) at $1.465 \mathrm{GHz}$ and $\mathrm{J} 2101+0341$ (right) at $1.465 \mathrm{GHz}$. 
S. Tinti et al.: High Frequency Peakers: Young radio sources or flaring blazars?, Online Material p 6

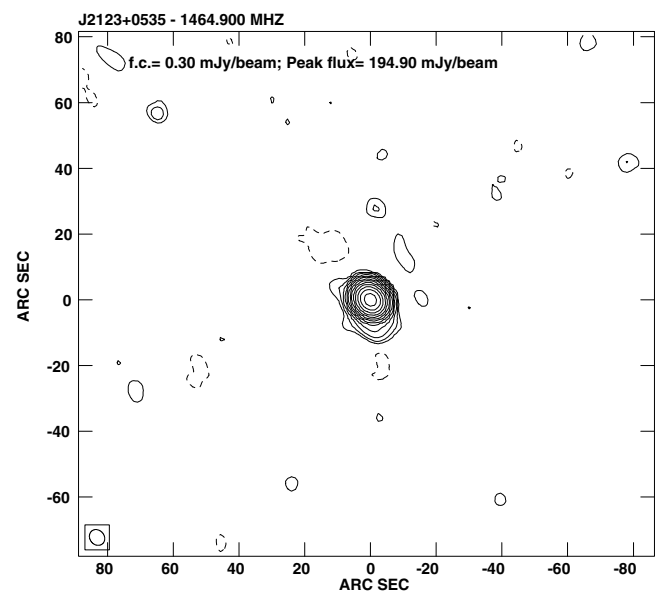

Fig. A.10. J2123+0535.
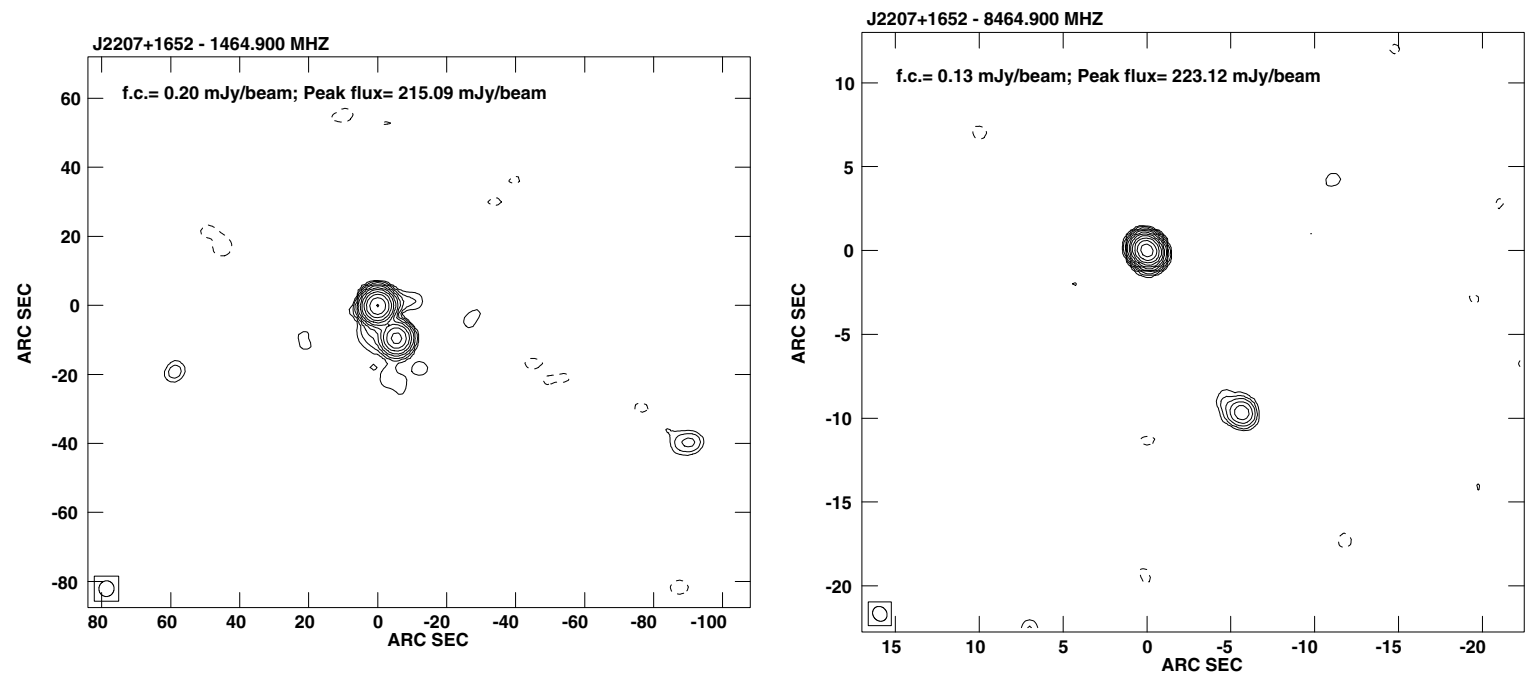

Fig. A.11. J2207+1652.
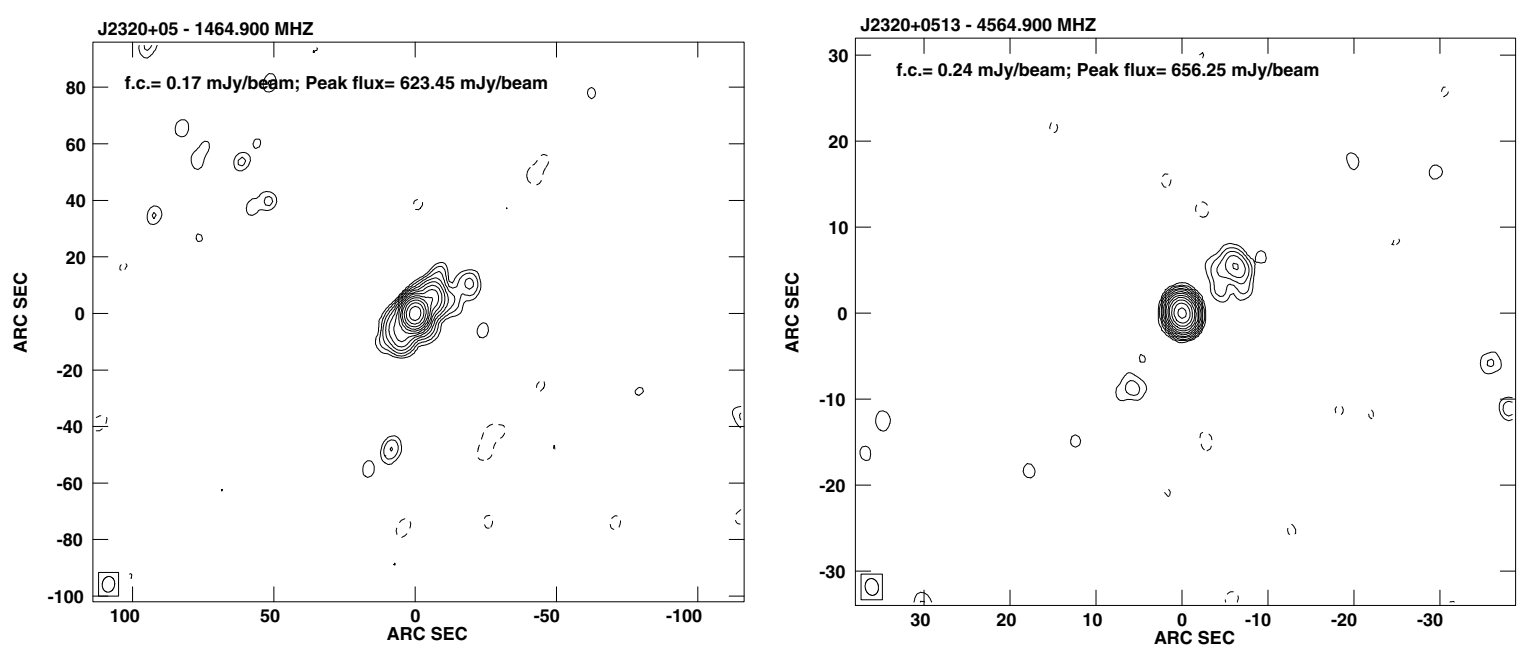

Fig. A.12. J2320+0513. 